\title{
Height formation of bright points observed by IRIS in Mg II line wings during flux emergence
}

\author{
M. Grubecka ${ }^{1}$, B. Schmieder ${ }^{2}$, A. Berlicki ${ }^{3,1}$, P. Heinzel ${ }^{3}$, K. Dalmasse ${ }^{4}$, and P. Mein ${ }^{2}$ \\ 1 Astronomical Institute, University of Wrocław, Kopernika 11, 51-622 Wrocław, Poland \\ e-mail: grubecka@astro.uni.wroc.pl \\ 2 LESIA, Observatoire de Paris, PSL Research University, CNRS, Sorbonne Universités, UPMC Univ. Paris 06, Univ. Paris Diderot, \\ Sorbonne Paris Cité, 5 place Jules Janssen, 92195 Meudon, France \\ e-mail: brigitte.schmieder@obspm.fr \\ 3 Astronomical Institute, Academy of Sciences of the Czech Republic, 25165 Ondrejov, Czech Republic \\ ${ }^{4}$ CISL/HAO, National Center for Atmospheric Research, PO Box 3000, Boulder, CO 80307-3000, USA
}

Received 14 September 2015 / Accepted 25 May 2016

\begin{abstract}
Context. A flux emergence in the active region AR 111850 was observed on September 24, 2013 with the Interface Region Imaging Spectrograph (IRIS). Many bright points are associated with the new emerging flux and show enhancement brightening in the UV spectra.

Aims. The aim of this work is to compute the altitude formation of the compact bright points (CBs) observed in Mg II lines in the context of searching Ellerman bombs (EBs).

Methods. IRIS provided two large dense rasters of spectra in $\mathrm{Mg} \mathrm{II} \mathrm{h}$ and $\mathrm{k}$ lines, $\mathrm{Mg}$ II triplet, $\mathrm{C} \mathrm{II}$ and $\mathrm{Si}$ IV lines covering all the active region and slit jaws in the two bandpasses (1400 ̊ and $2796 \AA$ ) starting at 11:44 UT and 15:39 UT, and lasting 20 min each. Synthetic profiles of Mg II and $\mathrm{H} \alpha$ lines are computed with non-local thermodynamic equlibrium (NLTE) radiative transfer treatment in 1D solar atmosphere model including a hotspot region defined by three parameters: temperature, altitude, and width.

Results. Within the two IRIS rasters, 74 CBs are detected in the far wings of the Mg II lines (at $+/-1 \AA$ and $3.5 \AA$ ). Around $10 \%$ of CBs have a signature in Si IV and CII. NLTE models with a hotspot located in the low atmosphere were found to fit a sample of Mg II profiles in CBs. The $\mathrm{H} \alpha$ profiles computed with these $\mathrm{Mg}$ II CB models are consistent with typical EB profiles observed from ground based telescopes e.g. THEMIS. A 2D NLTE modelling of fibrils (canopy) demonstrates that the Mg II line centres can be significantly affected but not the peaks and the wings of Mg II lines.

Conclusions. We conclude that the bright points observed in Mg II lines can be formed in an extended domain of altitudes in the photosphere and/or the chromosphere $(400$ to $750 \mathrm{~km}$ ). Our results are consistent with the theory of heating by Joule dissipation in the atmosphere produced by magnetic field reconnection during flux emergence.
\end{abstract}

Key words. line: profiles - Sun: chromosphere - Sun: activity - Sun: UV radiation - techniques: spectroscopic

\section{Introduction}

Emerging magnetic flux in the solar atmosphere is well observed in multi wavelengths using ground-based or space instruments (see the recent review of Schmieder et al. 2014). Signatures of magnetic flux emergence include, among others, intermittent brightenings in the wings of the $\mathrm{H} \alpha$ line $(+/-1 \AA$ to $10 \AA)$ the so-called Ellerman Bomb (EB; Ellerman 1917). The characteristics of these brightenings in optical wavelength range have been summarized by Georgoulis et al. (2002) after the Flare Genesis Experiment (FGE) observations and more recently by Rutten et al. (2013). EBs are observed as bright moustaches in chromospheric spectra owing to bright emission in the line wings. They have been observed in $\mathrm{H} \alpha$ (Kitai 1983), in Ca II $8542 \AA$ A, Ca H and K lines (Fang et al. 2006; Pariat et al. 2004, 2007; Watanabe et al. 2008, 2011; Hashimoto et al. 2010; Nelson et al. 2013a,b; Vissers et al. 2013). EBs commonly have a strong asymmetry in chromospheric lines and are also characterized by a deep absorption in the $\mathrm{H} \alpha$ line centre. Overlying arch filament system (AFS, canopy) may obscure the line centres and produce asymmetry of the core of the lines
(Rutten et al. 2013; Watanabe et al. 2011). Therefore, with some chromospheric observations of EBs, we can get information on heating only in the photosphere. This led to the conclusion of Rutten et al. (2013) that the hot plasma of EBs is located only in the deep photosphere. To understand the problem of canopy, a two cloud model was tested by Hong et al. (2014). This model shows the effects of the overlying cloud obscuring the line centre and the temperature variation of the second cloud, which models the atmosphere itself. However their fitting of the observed profiles depends on 11 parameters. Some of them have to be fixed under specific assumptions, which can influence the results.

EBs have an elliptical shape (Watanabe et al. 2011; Vissers et al. 2013; Nelson et al. 2015) and even jet-like or flame appearance in high-resolution images of the Swedish Solar Telescope (SST) in LaPalma or Hinode/SOT (Hashimoto et al. 2010). Their size depends on the wavelength indicating the existence of possible subcomponents (Hashimoto et al. 2010). The spatial movement of EBs could thus be interpreted by the successive emergence of subcomponents with different mass motions (Hashimoto et al. 2010; Hong et al. 2014). 
EBs are observed during the emergence of new active regions. Different magnetic configurations have been proposed for EBs during flux emergence (Bernasconi et al. 2002; Georgoulis et al. 2002; Matsumoto et al. 2008; Watanabe et al. 2011; Hashimoto et al. 2010; Nelson et al. 2013a,b; Vissers et al. 2013). Most of the EBs are associated with the inversion lines of small bipoles. Extrapolations suggest that the emerging loops have a sea-serpent shape with a succession of $U$ and $\Omega$ loops (Pariat et al. 2004). MHD simulations confirmed the undulatory characteristics of emerging loops and proposed that the local observed heating could be due to magnetic reconnection (Pariat et al. 2009; Isobe et al. 2007; Cheung et al. 2008; Archontis \& Hood 2009; Xu et al. 2011).

EBs have also been observed in EUV continua at $1600 \AA$ and $1700 \AA$, which are mainly formed in the low atmosphere, with TRACE and the Solar Dynamic Observatory (SDO/AIA) and show rapid variations of intensity (200 s) (Pariat et al. 2007; Vissers et al. 2013). The signature of EBs in the upper atmosphere is not clear. Pariat et al. (2007), Vissers et al. (2013) discussed on the observation of a EB related to a brightening observed in UV. This brigthening could correspond to the plasma of a surge-like event emitting in the C IV line at $1548 \AA$, which is also present in the $1600 \AA$ passband. The association of $\mathrm{H} \alpha \mathrm{EBs}$ and bright points at coronal temperatures in $171 \AA$ (TRACE, AIA) and in X-ray (Yohkoh/SXT, Hinode/XRT) is rare. In the data of FGE, only one bright feature was detected in $171 \AA$ (TRACE) and in X-ray and associated with one EB among the 47 identified EBs (Schmieder et al. 2004), and again it is possibly a jet.

The Interface Region Imaging Spectrograph (IRIS) with its high spatial resolution (pixel 0.167 arcsec, resolution of 0.3 arcsec), launched in June 2013 (De Pontieu et al. 2014) revealed the existence of bright points in the $\mathrm{Mg}$ II $\mathrm{h}$ and $\mathrm{k}$ chromospheric lines, and also in Si IV, CII and the Mg II triplet lines, which are formed in a wide range of temperatures (Peter et al. 2014; Vissers et al. 2015). These bright features are very intriguing because they are not visible in O IV lines and the Si IV profiles are blended by photospheric lines that are visible in absorption. Peter et al. (2014) called them "hot explosions in the cool atmosphere". Vissers et al. (2015) investigated the IRIS signatures of $\mathrm{H} \alpha$ EBs. Among the large number of detected $\mathrm{H} \alpha$ EBs in emerging regions, only a few of them were in the field of view of IRIS spectra and/or have an IRIS brightening counterpart signature.

Archontis \& Hood (2009) aimed at reproducing EBs via low atmospheric reconnection. The recent model of Archontis \& Hansteen (2014) is very promising for mimicking the hot bombs. However the current sheet where the reconnection can occur is located in the corona. This model as it is cannot really explain the lower atmospheric formation of the bombs described by Peter et al. (2014).

Thermodynamical models of EB spectra are based on NLTE radiative-transfer modelling in a simple 1D plane parallel semi-infinite atmosphere (Kitai 1983; Fang et al. 2006; Socas-Navarro et al. 2006; Herlender \& Berlicki 2011). Semiempirical models assume the hydrostatic equilibrium. However owing to readjustment of the kinetic temperature, heating is found not only in the photosphere, but also in the upper chromosphere. Recently Berlicki \& Heinzel (2014) constructed a grid of models based on a model of a quiet solar atmosphere and introduced a hotspot region at the photospheric level. By adjusting the four variable parameters for the hotspot (width, peak of temperature, density, position), they were able to fit the contrast of EBs observed in $\mathrm{Ca}$ II and $\mathrm{H} \alpha$ wings by the Dutch Open Telescope (DOT) instrument in LaPalma.

We had the opportunity to observe the emerging flux occurring in the AR NOAA11850 located at N08, E10 $(x=$ -310 arcsec, $y=100$ arcsec) on September 24, 2013 during the 60-day first joint observations of IRIS with the Télescope Héliographique pour l'Étude du Magnétisme et des Instabilités Solaires (THEMIS) magnetograph in Tenerife (Sect. 2), and the Multi-channel Subtractive Double Pass (MSDP) spectrograph (Mein \& Mein 1988) operating in the Meudon solar tower. Extended arcades of filaments called an Arch filament systems (AFS) overlying the region, direct signatures of an emerging flux, are well visible in MSDP H $\alpha$ and IRIS images (Fig. 1).

We focus our study on the Mg II, Si IV, and CII spectra observed with IRIS and $\mathrm{H} \alpha$ observed with THEMIS. The $\mathrm{H} \alpha$ observations are not co-temporal with IRIS observations and cannot be used to detect EBs directly as in the paper of Vissers et al. (2015). However we used the properties of the chromospheric EB spectra for the selection of the compact bright points (CB), which could possibly be recognized as an EB. EBs are perfectly detectable in the wings of chromospheric lines and are distinct from bright network points owing to their emission in a much wider wavelength domain, including far wings. Our selection of $\mathrm{CBs}$ is restricted to the field of view of flux emergence and is based on the brightening enhancements in the $\mathrm{Mg}$ II line wings by analogy to this chromospheric line behaviour in the visible range (Sects. 3 and 4). Tian et al. (2016) confirmed that the $\mathrm{Mg}$ II $\mathrm{k}$ and $\mathrm{h}$ lines can be used to investigate EBs that are similar to $\mathrm{H} \alpha$. With the 1D model of Berlicki \& Heinzel (2014) developed for $\mathrm{H} \alpha$, we compute the corresponding grid of profiles for $\mathrm{Mg}$ II lines (Sect. 5). We note that this model does not take into account the dynamics of the plasma and will therefore not explain the physics of the formation of the CBs. We compare the observed profiles with the synthetic profiles to determine the position and the extension of the hotspot in the temperature profile of the atmosphere, which could correspond to the heating that is due to magnetic reconnection (Sects. 6 and 7). We conclude on a large dispersion in these altitudes from the deep photosphere to the chromosphere (Sect. 8). We discuss the effects of introducing a cloud model by using a 2D NLTE modelling of fibrils. Our codes are limited to chromospheric temperatures and we have no information about higher reconnection altitudes in the atmosphere or heating to higher temperatures. The models proposed to fit the observed $\mathrm{Mg}$ II profiles produce $\mathrm{H} \alpha$ profiles that are consistent with the observations of THEMIS and the SST (Vissers et al. 2015).

\section{Observations}

\subsection{THEMIS}

The THEMIS instrument (López Ariste et al. 2000) was used in the Multi Temperature Raies (MTR) mode with two cameras. One camera recorded the four Stokes parameters in the $6302 \mathrm{Fe} \mathrm{I}$ line to do spectro-polarimetry and in the $\mathrm{H} \alpha$ line, i.e. see I+V and I-V image in Fig. 2 (top panel). We use the $\mathrm{H} \alpha$ spectra rastering the active region between 08:02 UT to 09:25 UT and 09:35 UT to 10:35 UT, which is just one hour before the IRIS observations. After removing the trend caused by instrumental effects, we calibrate the $\mathrm{H} \alpha$ spectra by using the David reference observed profiles (David 1961) following the method described in Berlicki et al. (2005). Firstly we determine a mean profile by averaging profiles along the slit containing an EB, but avoiding the EB profile. This mean profile is considered as our reference 

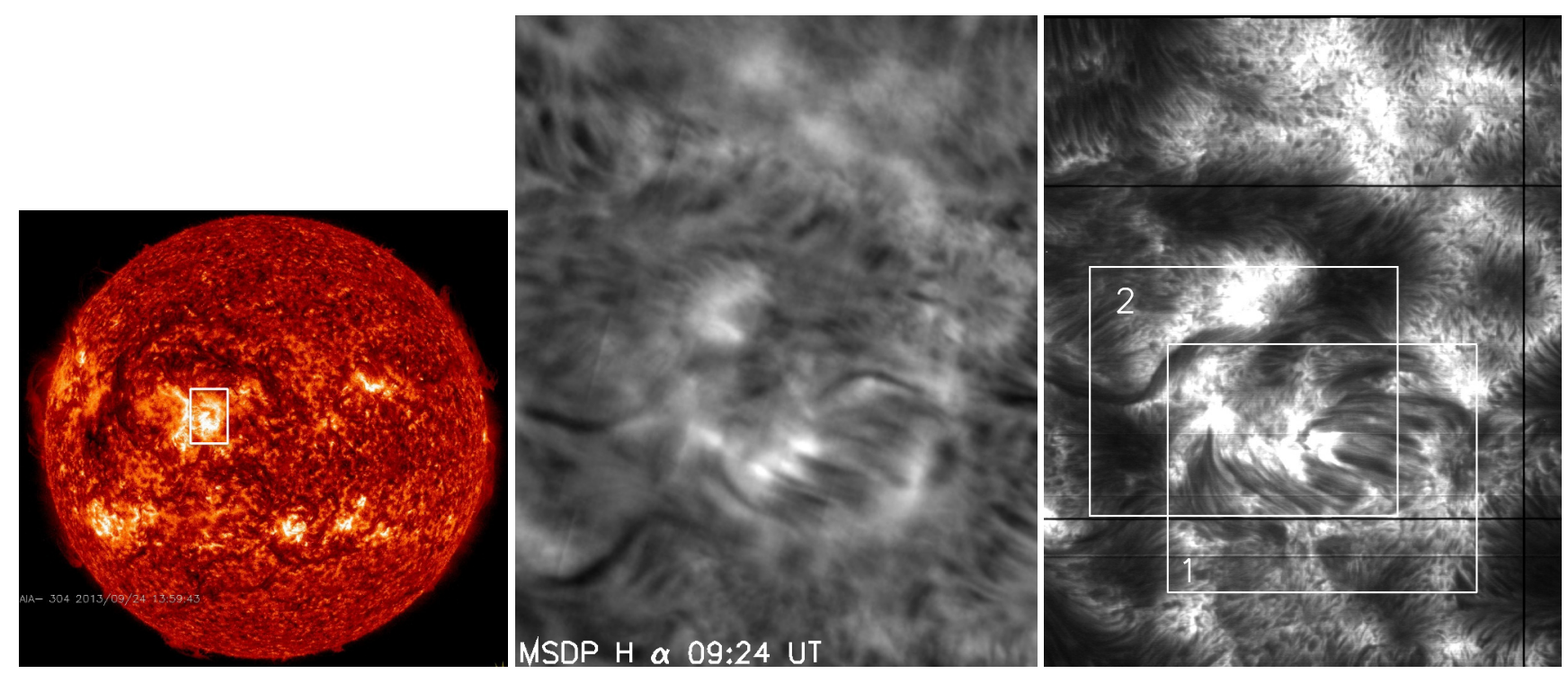

Fig. 1. Active region AR 11850 observed on September 24, 2013 from left to right: full disk image in AIA $304 \AA$, the white box indicates the field of view of the two right panels $\left(140 \times 175 \mathrm{arcsec}^{2}\right), \mathrm{H} \alpha$ image (MSDP/Meudon spectrograph) and Mg II k line centre at $2803.5 \AA$ obtained between 11:43 and 12:04 UT (IRIS spectrograph) showing the arch filament system (AFS). The two white boxes in the IRIS panel represent the field of view of the two small rasters presented in Figs. 3 and 4.

quiet Sun profile. We fit it with the David profile to obtain an absolute calibration, after taking into account the position of the slit in the Sun disk and the limb-darkening effect. The EB profile is calibrated in a second step with the same procedure. Figure 2 (bottom panel) presents the quiet Sun David profile, our reference quiet Sun profile, which fits well the David profile owing to its definition, the profile of one THEMIS EB, and the synthetic $\mathrm{H} \alpha$ profiles that we computed using the five NLTE models, each one determined by fitting an observed $\mathrm{Mg}$ II profile of a selected CBs (Sect. 9).

\subsection{IRIS}

IRIS performed two large dense rasters for $20 \mathrm{~min}$, each covering the whole active region from 11:43 UT to 12:04 UT and from 15:39 to 15:59 on September 24, 2013 (OBSID ref. 4000254145). The centre pointing of the rasters was $(x=$ -265.46 arcsec, $y=87.96$ arcsec $)$ for the first raster, $(x=$ -264.55 arcsec, $y=70.35$ arcsec) for the second raster. The spatial pixel size is 0.167 arcsec. Both rasters $\left(140 \times 175 \operatorname{arcsec}^{2}\right)$ consist of 400 spectra with a step size of 0.35 arcsec and taken with a cadence of less than 3 s. The rasters are obtained in a large number of lines in the near ultraviolet NUV window: 2783 to $2834 \AA$ and the two far ultraviolet windows: FUV1 1332-1348 $\AA$ and FUV2 1390-1406 $\AA$ wavelength bands. Table 1 gives the list of lines of interest for this study. During the 20 minutes of the raster acquisition, slit-jaw images (SJI) $\left(175 \times 175 \operatorname{arcsec}^{2}\right)$ were registered in the broadband filters (2796 $\AA$ and $1400 \AA$ ) with a cadence of 12 s and in $2832 \AA$ with a cadence of about $80 \mathrm{~s}$. The 1400 slit jaw intensity is an integration of the FUV emission within a range of about $55 \AA$, including the total emission of two Si IV 1402/1393 $\AA$ lines, the 2796 SJI intensity is integrated over $4 \AA$ around Mg II k $2796.35 \AA$ line. The Mg II h, and $\mathrm{k}$ are formed at chromosphere plasma temperatures $\left(10^{4} \mathrm{~K}\right)$. The ionization of the $\mathrm{Mg}$ II element occurs for a temperature equal to $2 \times 10^{4} \mathrm{~K}$. The SJI $2796 \AA$ filter gives images of the chromospheric temperature plasma. The $1400 \AA$ filter is a combination of the UV continuum formed at the temperature minimum and the emission of the Si IV lines formed at the transition region temperature. The co-alignment between the different channels is achieved by checking the position of the horizontal fiducial lines present in the spectra. Our main goal is to model the $\mathrm{Mg}$ II lines and compare them with the IRIS observations. We present the Si IV and CII spectra of the detected Mg II bright points for discussion.

\subsection{IRIS data radiometric calibration}

We downloaded level 2 data from the IRIS data base for our study (De Pontieu et al. 2014). IRIS spectra are given in the units of counts (DN). We calibrate the IRIS data based on the area response of the spectrograph from the preflight calibration (IRIS technical Note 24). We convert DN, after dividing it by the exposure time to observed intensity $\left(I_{\mathrm{obs}}[\mathrm{DN} / \mathrm{s}]\right)$ into physical units ( $\left.I_{\mathrm{abs}}\left[\mathrm{erg} \mathrm{s}^{-1} \mathrm{~cm}^{-2} \AA^{-1} \mathrm{sr}^{-1}\right]\right)$ in the following way:

$I_{\mathrm{abs}}=I_{\mathrm{obs}} \cdot k \cdot \frac{h c}{\lambda} \frac{1}{A_{\mathrm{eff}} \cdot d \cdot s a}$,

where $k$ is the number of photons per DN (4 in the case of NUV and 18 for FUV spectra). In our calculations, we have the following values: Planck constant $h=6.63 \times 10^{-27} \mathrm{erg}$, speed of light $c=3 \times 10^{10} \mathrm{~cm} \mathrm{~s}^{-1}, \lambda[\mathrm{cm}]$. The effective area $A_{\text {eff }}$ is available in SolarSoft through iris_get_response ()$, d=0.0254 \AA \times$ pixel $^{-1}$ is the dispersion. We need also the solid angle $s a$, which is

$s a=\frac{w \cdot p \cdot k^{2}}{d^{2}}$

where $w$ is the slit width $(0.33$ arcsec $), p$ pixel size along slit $(0.167 \operatorname{arcsec}), k(\mathrm{~km}$ per $\operatorname{arcsec}(727.1 \mathrm{~km})$, and $d$ distance to the Sun $\left(1.5 \times 10^{8} \mathrm{~km}\right)$. After calculation $s a$ is equal to $1.2949 \times$ $10^{-12} \operatorname{arcsec}^{2}$. To calibrate the data at Si IV 1394/1403 $\AA$ and C II $1336 \AA$, we used a similar procedure, but with different values of the effective area and dispersion. For Si IV $d=0.01298 \AA \times$ pixel $^{-1}$, for C II $d=0.01272 \AA \times$ pixel $^{-1}$. 
Table 1. IRIS lines of interest (rest wavelengths from the line list of Sandlin et al. 1986).

\begin{tabular}{|c|c|c|c|c|}
\hline $\begin{array}{l}\lambda \\
\AA\end{array}$ & $\begin{array}{l}\text { Width } \\
\AA\end{array}$ & Ion & Passband & Blends \\
\hline 1335.71 & 4.83 & $\mathrm{CII}$ & FUV1 & \\
\hline 1349.43 & 3.12 & Fe XII & FUV1 & \\
\hline 1355.66 & 4.36 & O I & FUV1 & \\
\hline 1393.76 & 5.46 & Si IV (1394) & FUV2 & S I (1392.59), Fe II (1392.82 and 1393.214), Ni II (1393.33) \\
\hline 1402.77 & 7.61 & Si IV (1403) & FUV2 & S I (1401.51), Fe II (1401.77-1403.10-1403.26) \\
\hline 2832.71 & 2.83 & continuum & NUV & \\
\hline 2814.45 & 3.67 & continuum & NUV & \\
\hline 2796.35 & 13.30 & Mg II k & NUV & Mn I lines \\
\hline 2797.9 & & $\mathrm{Mg}$ II (triplet1) & NUV & Fe I, Mn I lines \\
\hline 2798.0 & & $\mathrm{Mg}$ II (triplet2) & NUV & \\
\hline 2803.55 & 13:30 & $\mathrm{Mg}$ II h & NUV & \\
\hline
\end{tabular}

\section{Compact brightenings in Mg II lines}

\subsection{Mg II spectroheliograms}

From the two recorded rasters of IRIS spectra, we create spectroheliograms of the active region in $\mathrm{Mg}$ II $\mathrm{h}$ line at specific wavelengths: $\Delta \lambda=-3.5 \AA, \Delta \lambda=0 \AA, \Delta \lambda=+0.23 \AA$, $\Delta \lambda=+1 \AA$ (Figs. 3 and 4 ) to visually detect CBs, which we examine in the context of searching EBs. Therefore we reduce the size of the studied areas to two smaller regions concerned only with the flux emergence, which were recorded in $11.5 \mathrm{~min}$ from 11:49-12:00 UT (raster 1) and from 15:46-15:58 UT (raster 2) (white boxes in Fig. 1 right panel), regions where the AFS are well visible in $\mathrm{H} \alpha$ and $\mathrm{Mg}$ II h3 line centre images (Fig. 1). Each $\mathrm{CB}$ emission covers 1 to 6 pixels along the slit as is shown in the top panels in Fig. 5. Figure 6 (bottom left panel) gives an example of three different profiles in the CB (B). The adjacent spectra at $+/-0.35$ arcsec did not show a clear enhancement of emission. This indicates that the size of the $\mathrm{CB}$ is around one arcsec. Comparing the spectroheliograms of these two smaller regions shown in Figs. 3 and 4, we note the difference of appearance of CBs according to their wavelength. At $\lambda=0.23 \AA$, which represents the common position of the Mg II h peaks (h2v, h2r), AFS and some bright CBs are observed. More CBs are detected in the wings of the $\mathrm{Mg}$ II lines, i.e. at $\Delta \lambda=-3.5 \AA$ (top panel) and $\Delta \lambda=+1 \AA$ (middle panel). The brightenings of some CBs are, nevertheless, very weak in the far wings and not detectable in SJI $2796 \AA$ because of the low intensity in their peaks, h2r and $h 2 \mathrm{v}$. The contribution of the emission of the peaks of the near-by chromosphere can be more important than the increase of emission in the wings in these CBs and diminish the contrast of the CBs in the SJIs, which are recorded in a relatively small wavelength band pass ( $4 \AA$ ) centred on the peaks compared to the broad Mg II k line extension over $13 \AA$. These CBs detected in the far wings do not always have a corresponding $\mathrm{CB}$ detectable in $\mathrm{h} 2 \mathrm{v}-\mathrm{h} 2 \mathrm{r}$ as we will discuss in the examples in Sect. 4.2. We decided to analyze mostly the $\mathrm{Mg}$ II $\mathrm{h}$ line (and not $\mathrm{Mg}$ II k) in the spectra because of the IRIS spectral window range is limited in the blue wing of $\mathrm{Mg}$ II k line at $-2 \AA$ from the $k$ line centre, while the red wing of h line is observed up to $4.5 \AA$. Then the modelling of the Mg II h line can be completed in its far red wing. This is better for the comparison between synthetic and observed profiles (Sects. 5 and 6). In Fig. 3, we add the spectroheliogram obtained in the continuum at $+26.6 \AA$ of the $\mathrm{Mg}$ II h line centre. Some bright points in the continuum are still visible.
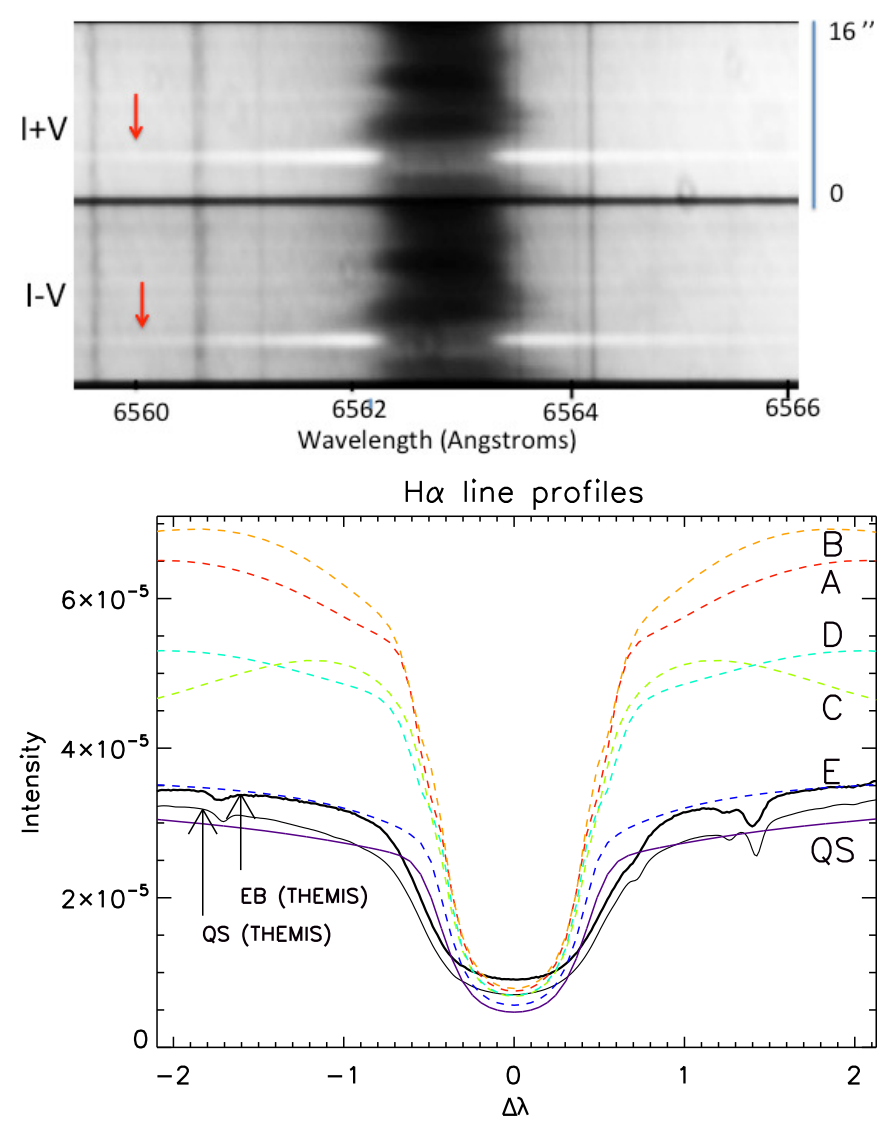

Fig. 2. Top panel: spectrum of an EB in $\mathrm{H} \alpha$ line observed by THEMIS/MTR spectropolarimeter. The two red arrows indicate the EB in $\mathrm{I}+\mathrm{V}$ and $\mathrm{I}-\mathrm{V}$ channels. Bottom panel: $\mathrm{H} \alpha$ profiles - Observed profiles: EB profile of the THEMIS spectrum shown in the top panel (solid black line), observed nearby quiet Sun profile from THEMIS (thin black line). Synthetic profiles computed from NLTE modelling: QS (purple solid line). All colour profiles described with letters from A to E are calculated for each best model of selected CBs (A - red; B - orange; $\mathrm{C}$ - light green; $\mathrm{D}$ - light blue; $\mathrm{E}-$ dark blue). Intensity units is $\left(\operatorname{erg~s}^{-1} \mathrm{~cm}^{-2} \AA^{-1} \mathrm{sr}^{-1}\right), \Delta \lambda$ unit is $\AA$.

Since our radiative models used as the basis for the spectral synthesis cannot emulate dynamics, we select the less asymmetric profile in each $\mathrm{CB}$. Figure 6 (left bottom panel) gives an example of our selection for the CB (B) which presents emission in six pixels along the slit for one spectra. B2, B3 pixels have asymmetric profiles in $\mathrm{Mg}$ II $\mathrm{h}$ line and in Si IV lines. We choose 
M. Grubecka et al.: Bright points in Mg II lines

AR1 1850 at $\Delta \lambda=-3.5 \AA$ from Mgll $h$ line center, 11:49-12:00 UT

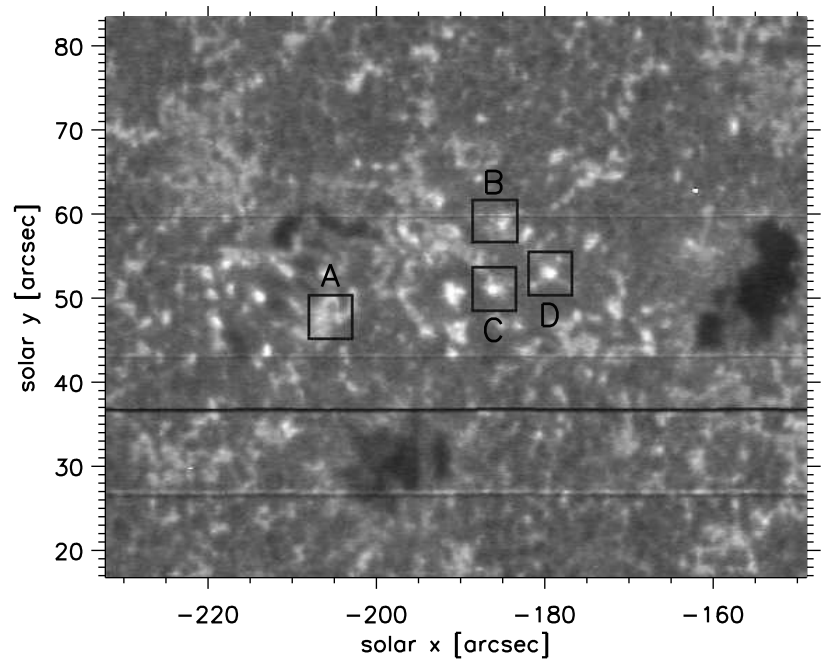

AR1 1850 at $\Delta \lambda=+0.23 \AA$ from Mgll $h$ line center, 11:49-12:00 UT

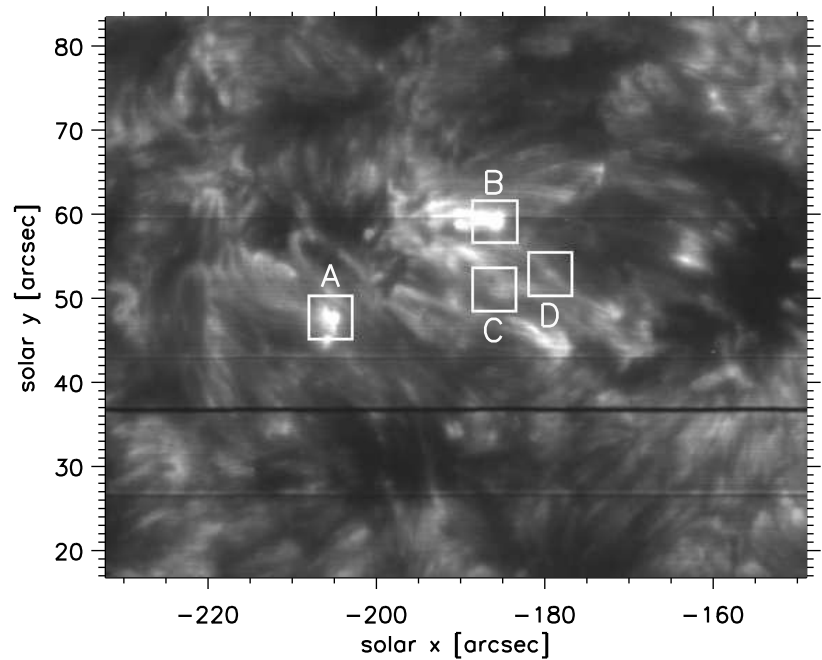

AR11850 at $2831.1 \dot{A}, 11: 49-12: 00$ UT

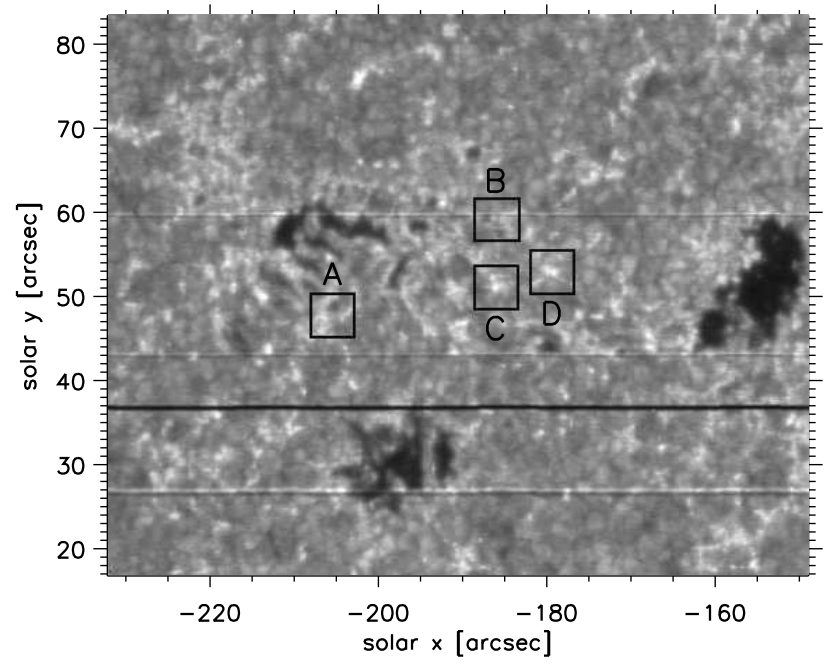

AR11850 at Mgll $h$ line center, 11:49-12:00 UT

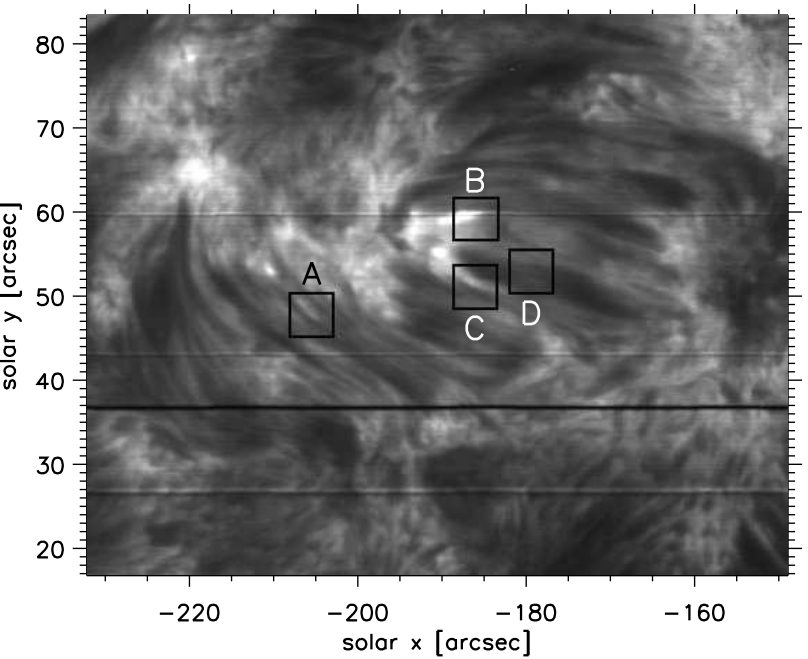

AR1 1850 at $\Delta \lambda=+1.0 \AA$ from Mgll $h$ line center, 11:49-12:00 UT

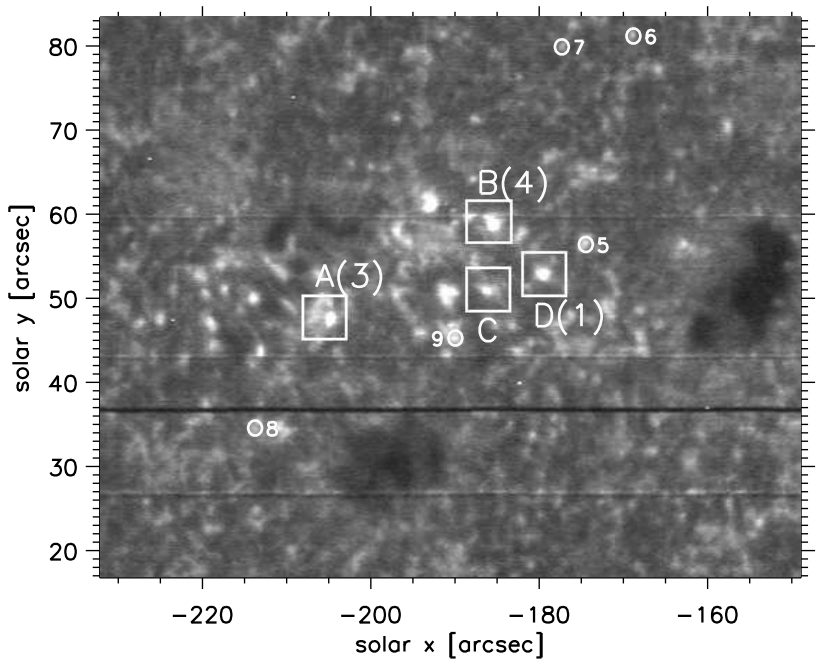

AR11850 at Si IV 1402.8 $\dot{A}, 11: 49-12: 00$ UT

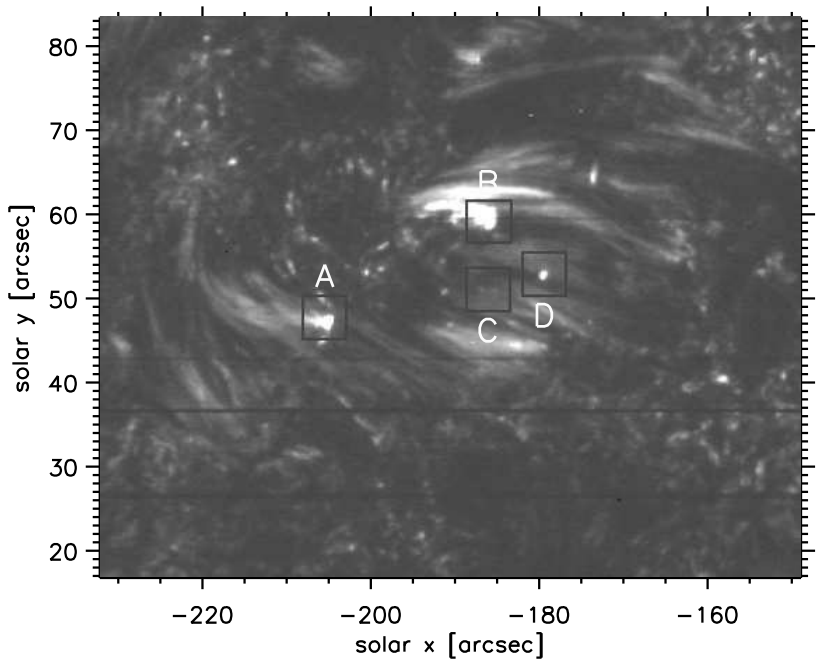

Fig. 3. AR 11850 IRIS observations of raster 1 (the field of view is indicated in Fig. 1, right panel). Top and middle panels from left to right: Mg II spectroheliograms at $2800.0 \AA, 2803.5 \AA$, $2803.7 \AA$ and $2804.5 \AA$. Bottom left panel: spectroheliogram in the continuum at $2831.1 \AA$ A Bottom right panel: spectroheliogram in Si V line. White or dark boxes show the selected CBs from A to D (the numbers in parenthesis indicate the hot explosions of Peter et al. 2014), small white circles with numbers 5 to 9 in the second row, right panel are examples of small brightenings. 
AR11850 at $\Delta \lambda=-3.5 \AA$ from Mgll $h$ line center, $15: 46-15: 58$ UT

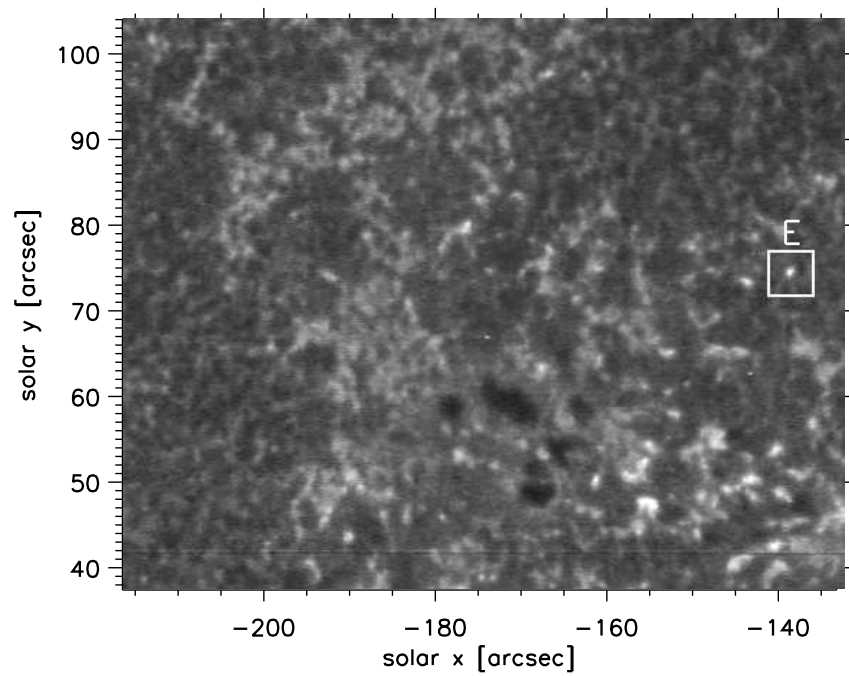

AR11850 at Mgll $h$ line center, 15:46-15:58 UT

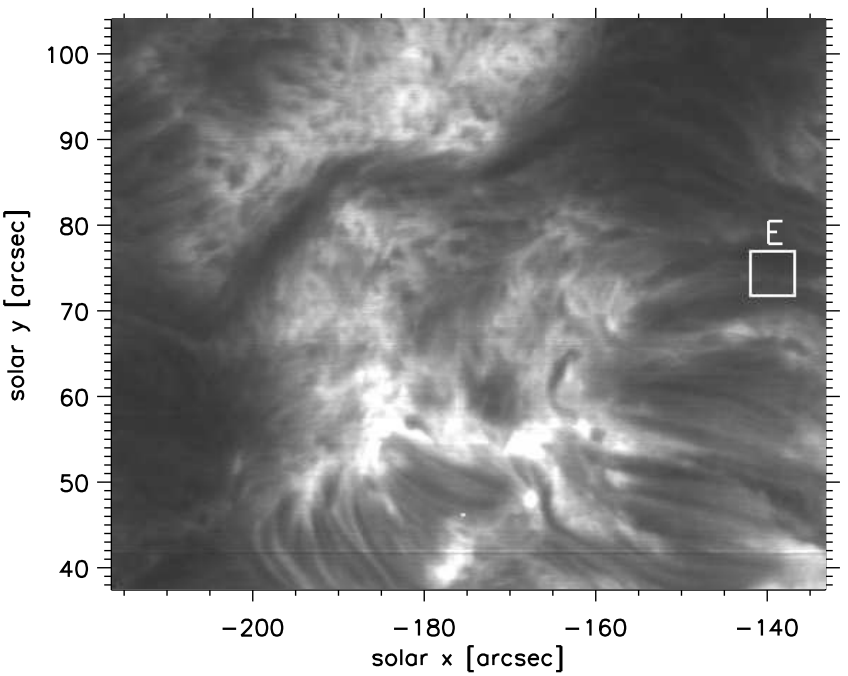

AR11850 at $\Delta \lambda=+0.23 \AA$ from Mgll $h$ line center, $15: 46-15: 58$ UT AR11850 at $\Delta \lambda=+1.0 \AA$ from Mgll $h$ line center, 15:46-15:58 UT

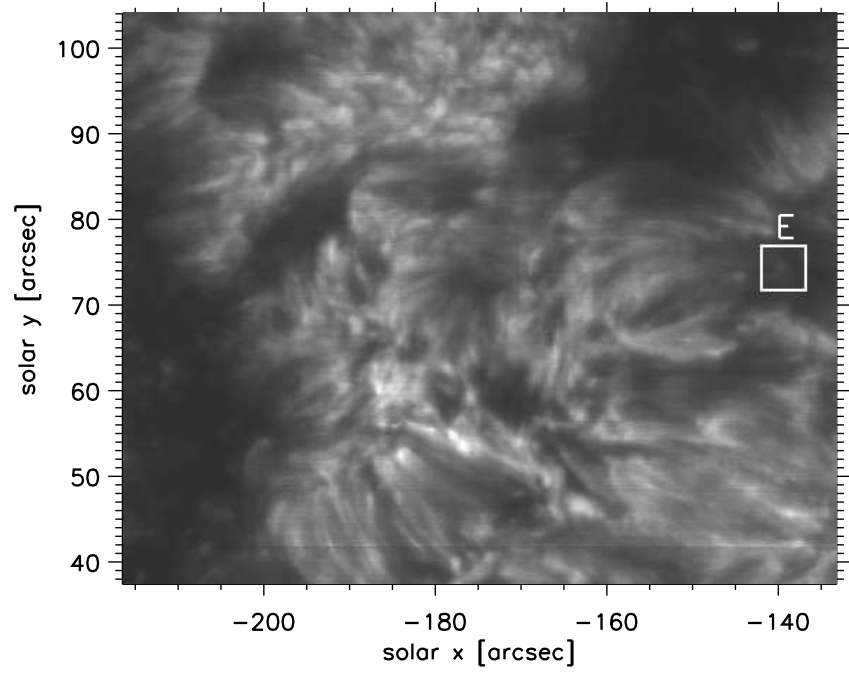

AR11850 at Si IV $1402.8 \AA$

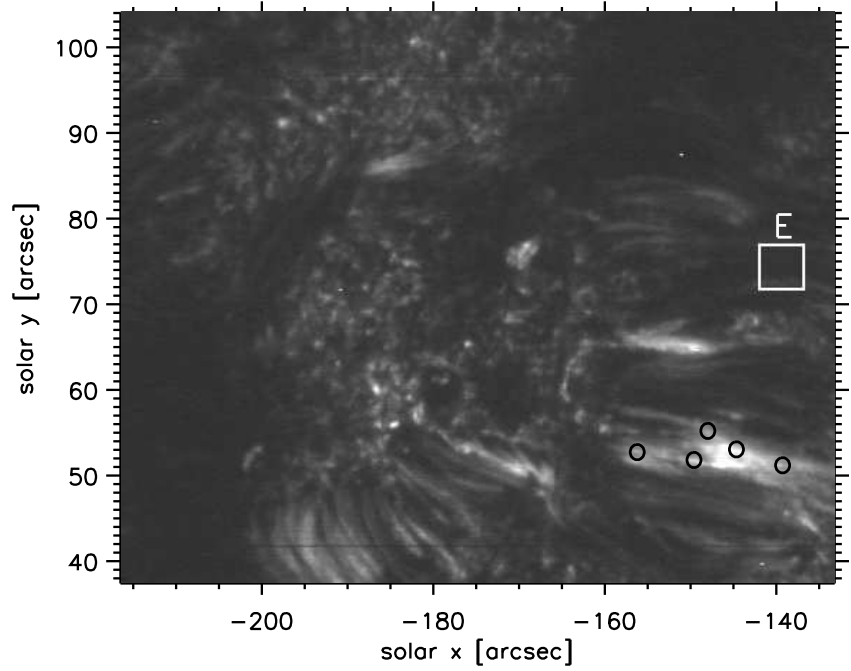

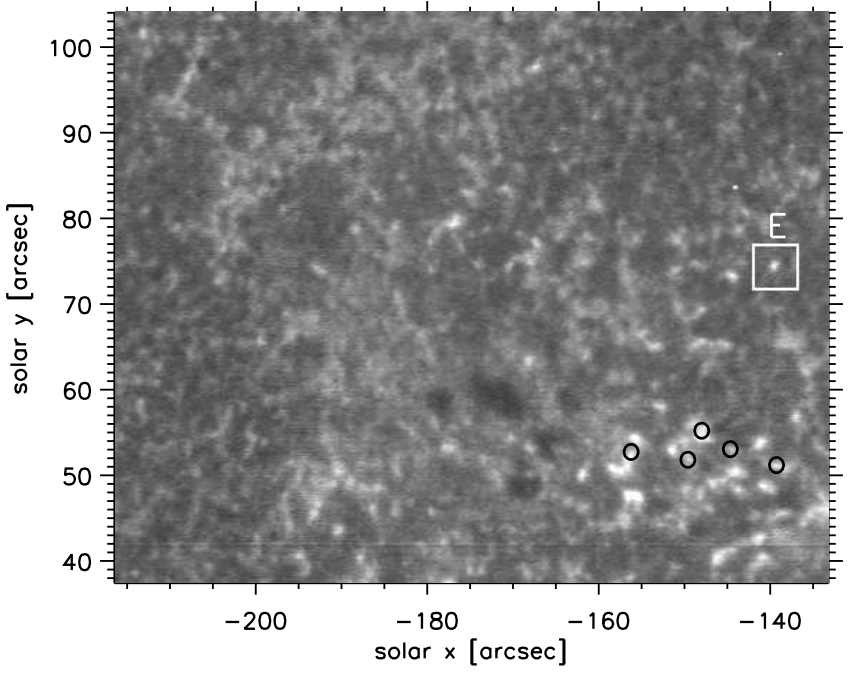

Slit-jaw image $2796 \AA$ A $24.09 .13 \quad 15: 51: 55 \mathrm{t}=59$

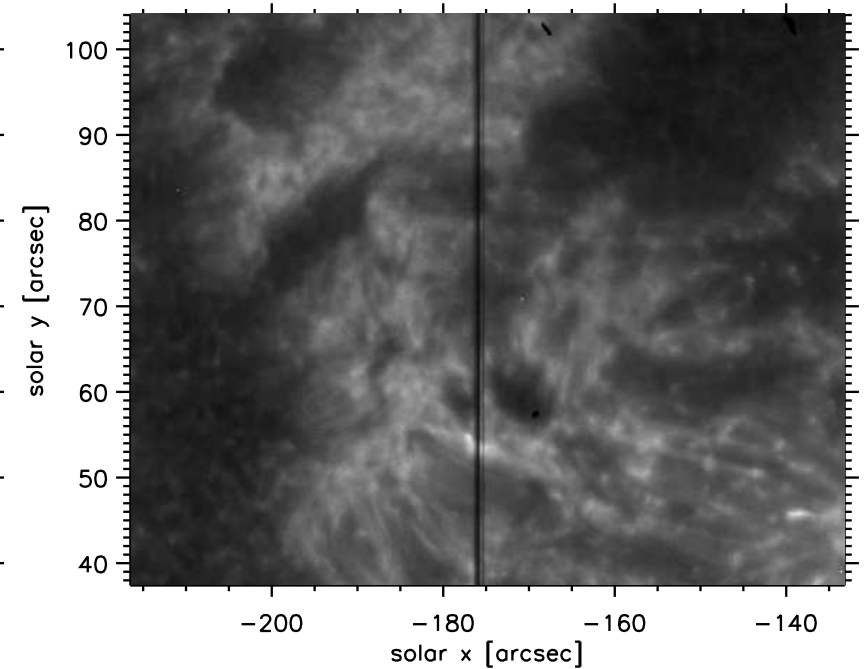

Fig. 4. AR 11850 IRIS observations of raster 2 (the field of view is indicated in Fig. 1, right panel). Top and middle panels from left to right:

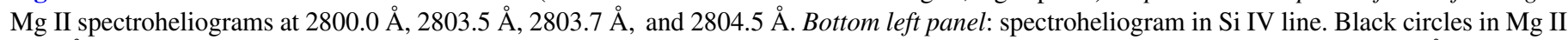
$+0.1 \AA$ and in Si IV spectroheliogram indicate CBs visible in Si IV and Mg II line wing. Bottom right panel: slit-jaw image at $2796 \AA$. The white box indicates the location of the CB (E) that we have analysed. The EB (E) is not visible in Si IV and Mg II core of the line. 
B1 for the CB (B). This profile corresponds generally to a pixel in the central part of the $\mathrm{CB}$.

\subsection{Statistics of the bright points}

We count the compact bright points in the restricted emerging area where AFS are visible. For raster 1, $42 \mathrm{CBs}$ are identified in the image in $\mathrm{Mg}$ II $\mathrm{h}$ line at $\Delta \lambda=-3.5 \AA$ with a contrast better than 2 , while only 38 of these $42 \mathrm{CBs}$ are still visible in the image at $\Delta \lambda=+1 \AA$. For raster 2 , the numbers are similar $(36 \mathrm{CBs})$ if we take into account that a part of the emerging region is cut. The global number of CBs in the two rasters is 74 .

Let us see how many are detected in hot temperature lines. In the slit jaw images at $1400 \AA$ corresponding to the field of view of raster 1, only four brightenings are identified (see Fig. 1 in Peter et al. (2014). For the field of view of raster 2, the number of brightenings in spectroheliogram at $1402.8 \AA$ and in SJI 2796 is difficult to be evaluated owing to the bright elongated structures but can be estimated as 4 to 5 . They correspond to the CBs observed in the wing of $\mathrm{Mg}$ II at $1 \AA$ (Fig. 4 bottom left panel and middle right panel). The emerging flux is between $x=-160$ and $x=-135$ and $y=38$ to 80 arcsec. Four brightenings are detectable but mixed with bright fibrils overlying the region. Finally among the $74 \mathrm{CBs}$ detected in $\mathrm{Mg}$ II lines, only eight brightenings have a signature in hot lines ( $\mathrm{Si}$ IV and CII). We conclude that the number of bright points in SJI images is about ten times smaller than the detected Mg II CBs.

After looking at the Fig. 1 of Vissers et al. (2015), comparing $\mathrm{H} \alpha$ EBs and bright features visible in Si IV and CII lines, we can estimate a similar result. This should be checked certainly in more details.

Finally, we focus our study on five selected CBs which are representative of the full sample. All the CBs that we have studied are observed in the far wings of $\mathrm{Mg}$ II lines because of our selection criteria. Only a few of them $(10 \%)$ have large intensity peaks or very wide profiles. The selected CBs are shown in Figs. 3 and 4 (white boxes indicated by the letters A to E). The CBs (A) and (B) are the best visible in the Mg II spectroheliograms at $\Delta \lambda=+0.23 \AA$ (peaks) and $\Delta \lambda=+1.0 \AA$ (Fig. 3). We can hardly see that the CBs (A) and (B) in the far wings $(\Delta \lambda=-3.5 \AA)$. The $\mathrm{CB}(\mathrm{C})$ is not visible in the panels at $\mathrm{Mg}$ II line centre (the bright line could be due to a bright fibril) and in the peaks. The CB (D) are visible in each panel in Fig. 3, except in the panel at $\mathrm{Mg}$ II $\mathrm{h}$ line centre where a dark fibril is instead observed. The CB (E) is visible in the spectroheliograms obtained in the far wing of $\mathrm{Mg}$ II line at $\Delta \lambda=+1 \AA$ and $\Delta \lambda=-3.5 \AA$ (Fig. 4).

Three of the selected CBs have a signature in the Si IV spectroheliogram (Figs. 3 bottom right panel). The CBs named A, $\mathrm{B}$, and $\mathrm{D}$ correspond to the hot explosions 3,4 , and 1 , respectively cited in Peter et al. (2014). The other two CBs, C and E, have no signatures in Si IV spectroheliograms. Our main goal is to find and determine which CBs visible in $\mathrm{Mg}$ II lines could correspond to EBs by also modelling the $\mathrm{H} \alpha$ line with the same atmosphere models. We perform a radiometric calibration of the $\mathrm{Mg}$ II spectra, which has not been achieved earlier for this data set in order to compare the observed profiles with synthetic profiles computed from NLTE models.

\section{IRIS spectra and profiles of the CBs}

For the five selected CBs, we present the concerned $\mathrm{Mg}$ II spectra along the slit and point by an arrow the pixel inside the $\mathrm{CB}$
(A to E) (Fig. 5 top panels). The corresponding profiles are presented in each panel in Fig. 5 bottom panels, and all are summarized in the bottom right panel to see their differences in one glance. The $\mathrm{Mg}$ II $\mathrm{h}$ and $\mathrm{k}$ lines and the two lines of the $\mathrm{Mg}$ II triplet between them are observed in a unique spectral window of IRIS around $13 \AA$ wide. In the same way, we present the $\mathrm{SiV}$ and CII line spectra along the slit and profiles of the five pixels, one or two CBs are in each spectra (Fig. 6).

\subsection{Quiet Sun profiles}

The quiet Sun profile (QS) (average profile from IRIS observations of disk centre at 04:20 UT) and a local quiet Sun profile $\left(\mathrm{QS}_{\mathrm{loc}}\right)$, obtained by averaging 20 profiles of $\mathrm{Mg}$ II $\mathrm{h}$ spectra observed out of the $\mathrm{CB}$ along the same slit position, are also presented in Fig. 5. The QS profile is very similar to the Mg II profile determined by Staath \& Lemaire (1995). The QS profile of $\mathrm{Mg}$ II lines consists of a large absorption profile of more than $20 \AA$ wide with two pairs of emission peaks, one for $h$ and one for $\mathrm{k} \mathrm{Mg}$ II lines, in the central part of the absorption profile. The IRIS window (13 $\AA$ ) is centred in this absorption profile in the middle of the $\mathrm{Mg}$ II $\mathrm{h}$ and $\mathrm{k}$ lines. In the following work we concentrate on the $\mathrm{Mg}$ II h line which, in our observations, presents a more extended red wing than the blue wing of $\mathrm{k}$. The two peaks of the $\mathrm{h}$ line have a maximum of intensity of $4 \times 10^{5} \mathrm{erg} \mathrm{s}^{-1} \mathrm{~cm}^{-2} \AA^{-1} \mathrm{sr}^{-1}$, a FWHM of $0.2 \AA$, the centre of the line an intensity value of $1.5 \times 10^{5} \mathrm{erg} \mathrm{s}^{-1} \mathrm{~cm}^{-2} \AA^{-1} \mathrm{sr}^{-1}$, and the separation of the two peaks a distance of $0.4 \AA$. The mean profiles of the $\mathrm{Mg}$ II lines close by the $\mathrm{CBs}\left(\mathrm{QS}_{\text {loc }}\right)$ have the same absorption profile in the IRIS window, but slightly higher intensity in the peaks and in the line centre than the QS. The FWHM of the peaks and their separation are unchanged.

\subsection{Characteristics of the $C B$ line profiles}

The global shape of $\mathrm{Mg}$ II $\mathrm{k}$ and $\mathrm{k}$ lines is similar in CBs, as can be checked in Fig. 5. We focus our analysis on the Mg II h line profile because of its extending red wing, as we explained above. The line profiles of (A) and (B) have a strong Mg II peak intensity ( $\mathrm{h} 2 \mathrm{v}$ and $\mathrm{h} 2 \mathrm{r}$ ). Their intensity is by one order of magnitude higher than the peaks of the QS profile and by a factor 2 with the peaks of $\mathrm{QS}_{\text {loc }}$ (Fig. 5, middle panels). Their FWHM is of the order of $1.5 \AA$. The centre intensity h3 in the $\mathrm{Mg}$ II h line for (A) is comparable to $\mathrm{h} 3$ intensity of $\mathrm{QS}_{\text {loc }}$ profile. Its value is around $1 \times 10^{5} \mathrm{erg} \mathrm{s}^{-1} \mathrm{~cm}^{-2} \AA^{-1} \mathrm{sr}^{-1}$. On the contrary h3 for B is increased to $2.1 \times 10^{5} \mathrm{erg} \mathrm{s}^{-1} \mathrm{~cm}^{-2} \AA^{-1} \mathrm{sr}^{-1}$. We note that (A) and (B) have a strong emission in Si IV, CII lines (Fig. 6).

For (C), the $\mathrm{Mg}$ II h spectrum presents an emission enhancement in the two peaks particularly in $h 2 v$, but the values of the emission do not reach the h2 values of (A) and (B) spectra. Additionally a strong emission enhancement is noted in the far wings (Fig. 5). The emission is over the whole wavelength range. The h3 emission reaches nearly $1.5 \times 10^{5} \mathrm{erg} \mathrm{s}^{-1} \mathrm{~cm}^{-2} \AA^{-1} \mathrm{sr}^{-1}$, which is more than the $\mathrm{QS}_{\text {loc }}$. The $\mathrm{Mg}$ II h profile of (C) presents a strong peak asymmetry, which indicates the presence of either material flows or overlying dynamical fibrils. The $\mathrm{CB}(\mathrm{C})$ has no counterpart in Si IV and CII spectra (Fig. 6). However the $\mathrm{Mg}$ II triplet intensity in (C) is enhanced and shows the same asymmetry as the $\mathrm{Mg}$ II $\mathrm{h}$ and $\mathrm{k}$ lines.

The (D) brightening, according to Table 2 is, in many respects, similar to the $\mathrm{C}$ brightening, however it reveals certain differences owing to the raggedness? of the D profile, for 
Spectrum of CB A, 11:53:11

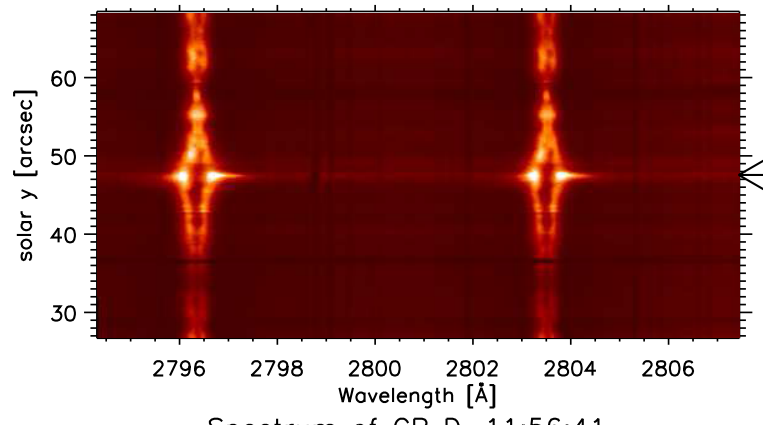

Spectrum of CB D, 11:56:41

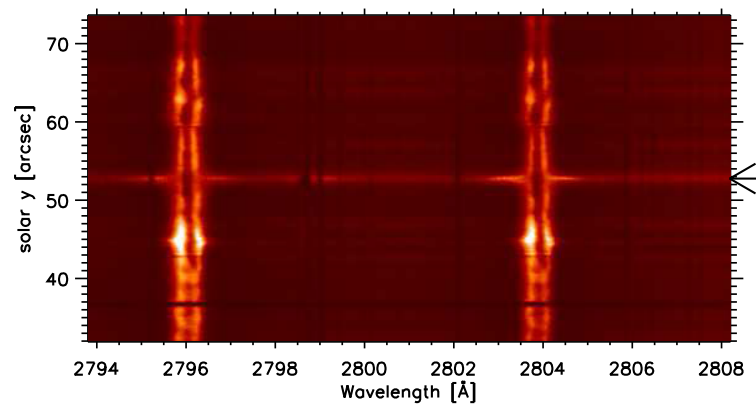

$\mathrm{CB} \mathrm{A}, \mathrm{Mg} \| \mathrm{h}$ and $\mathrm{k}$ lines

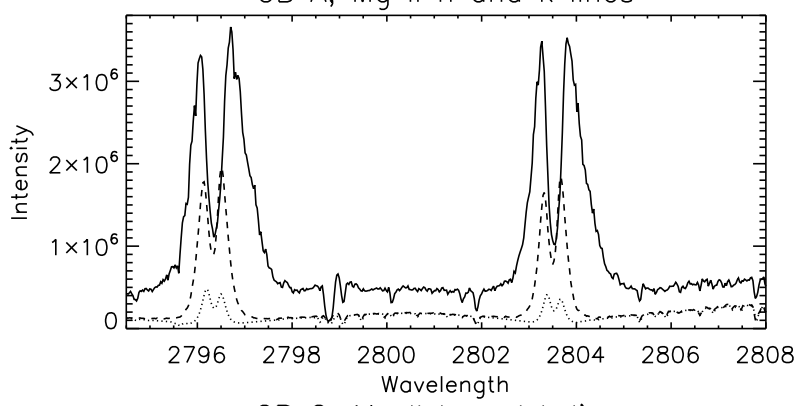

$\mathrm{CB} \mathrm{C}, \mathrm{Mg} \| \mathrm{h}$ and $\mathrm{k}$ lines

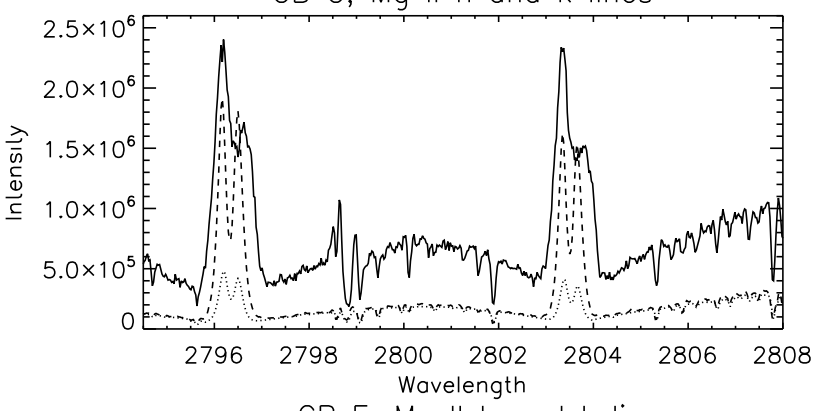

$\mathrm{CB} \mathrm{E}, \mathrm{Mg} \| \mathrm{h}$ and $\mathrm{k}$ lines

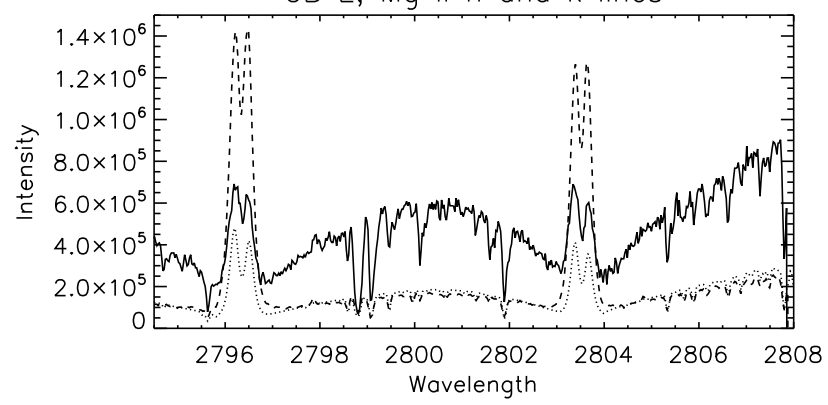

Spectrum of CBs B and C, 11:55:45

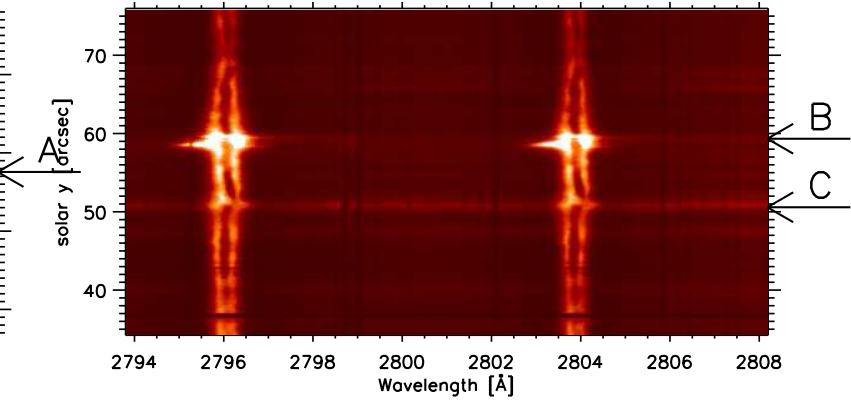

Spectrum of CB E, 15:57:07

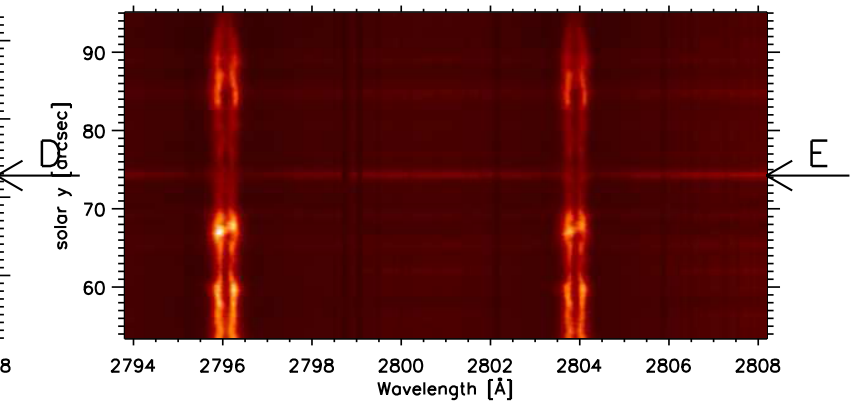

$\mathrm{CB} \mathrm{B}, \mathrm{Mg} \| \mathrm{h}$ and $\mathrm{k}$ lines

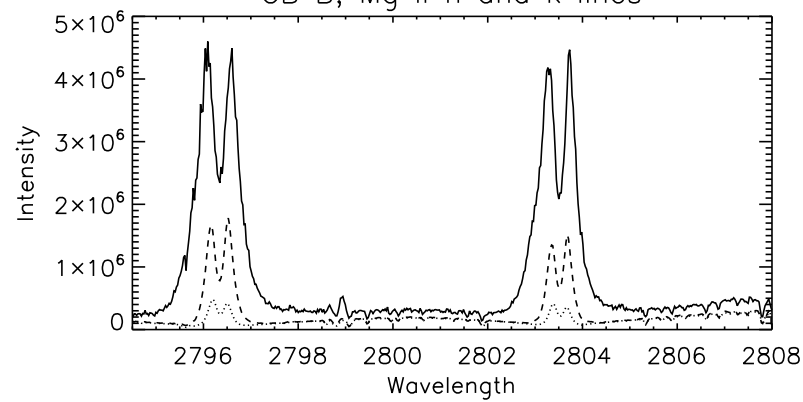

CB D, $M g \| h$ and $k$ lines
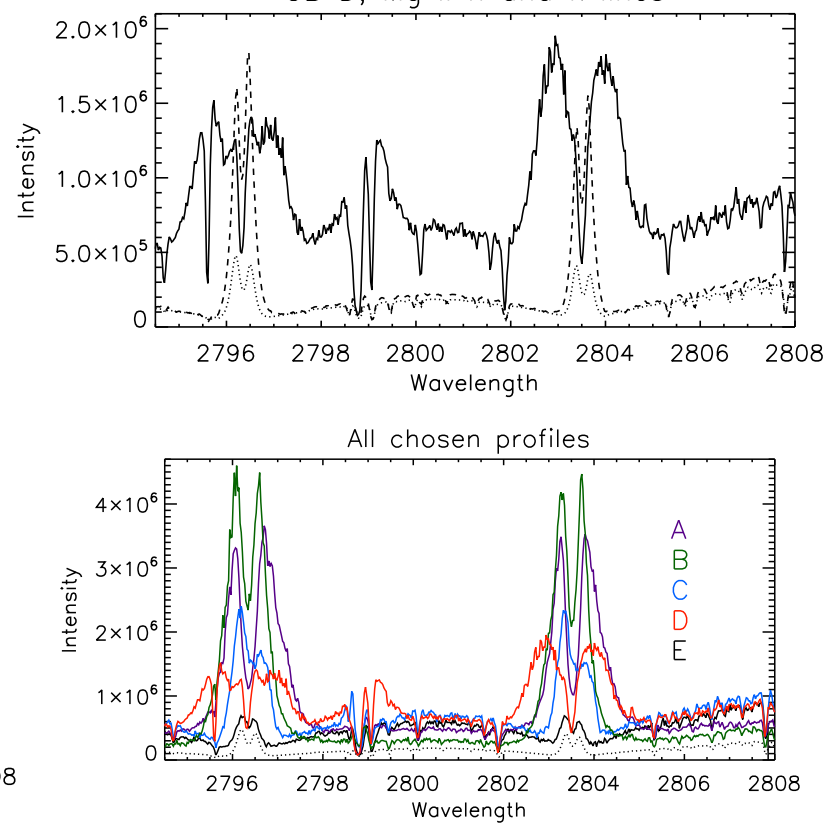

Fig. 5. Spectra and profiles of Mg II h and k and Mg II triplet at $2798.7 \AA$ lines for the five selected CBs. Two top rows: spectra of the CBs shown in the boxes of Figs. 3 and 4. The arrows indicate the positions of each CB: A to E at $y=47.5,59.0,50.9,52.7,74.2$ arcsec, respectively, where the profiles are taken and drawn below. Bottom five plots: profiles of A to E (solid line) with the QS (dotted line) and the nearby quiet profile (dashed thin line). Bottom right panel: plot of all the selected profiles shown in a different colour: A - purple; B - green; C - blue; D - red; and E - black. Intensity units is (erg s${ }^{-1} \mathrm{~cm}^{-2} \AA^{-1} \mathrm{sr}^{-1}$ ), wavelengths unit is $\AA$. For CBs C and D, we note that the emission of Mg II triplet is enhanced and its line profile is absorbed by an Mg II line (2798.6 $\AA$ ) in the blue wing and by an Mn I line (2799.2 $\mathrm{A})$ in the red wing. 
Si IV $1394 \AA$

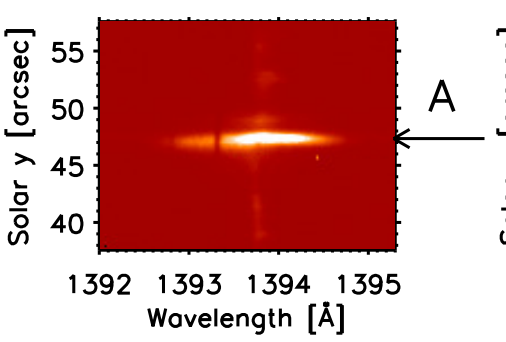

Si IV $1394 \AA$
Si IV $1403 \AA$

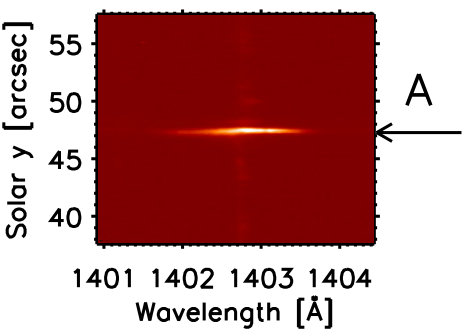

Si IV $1403 \AA$
C II $1336 \AA$

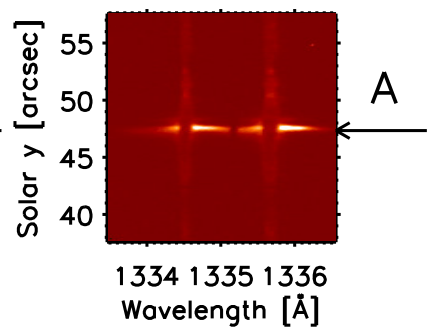

C II $1336 \AA$

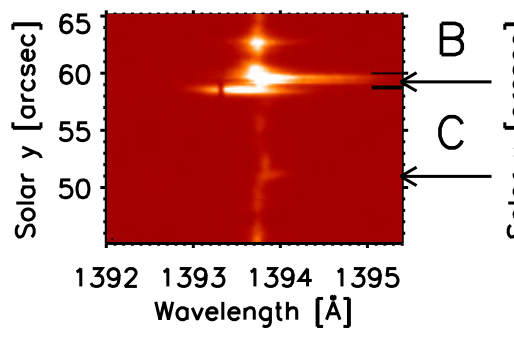

Si IV $1394 \AA$
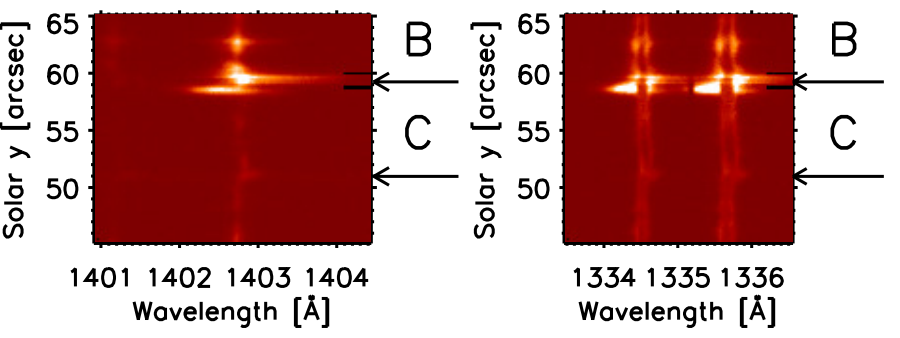

Si IV $1403 \AA$

C II $1336 \AA$

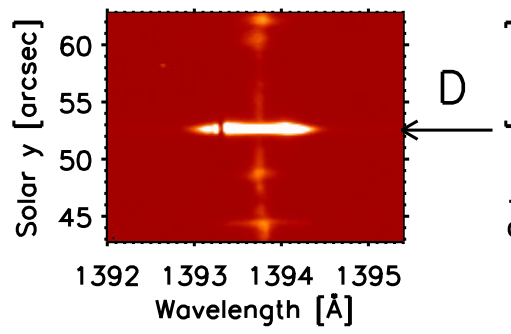

Si IV $1394 \AA$
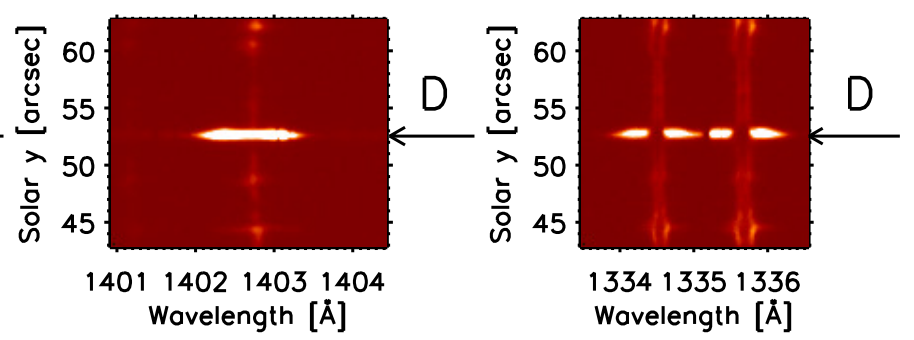

Si IV $1403 \AA$

C II $1336 \AA$
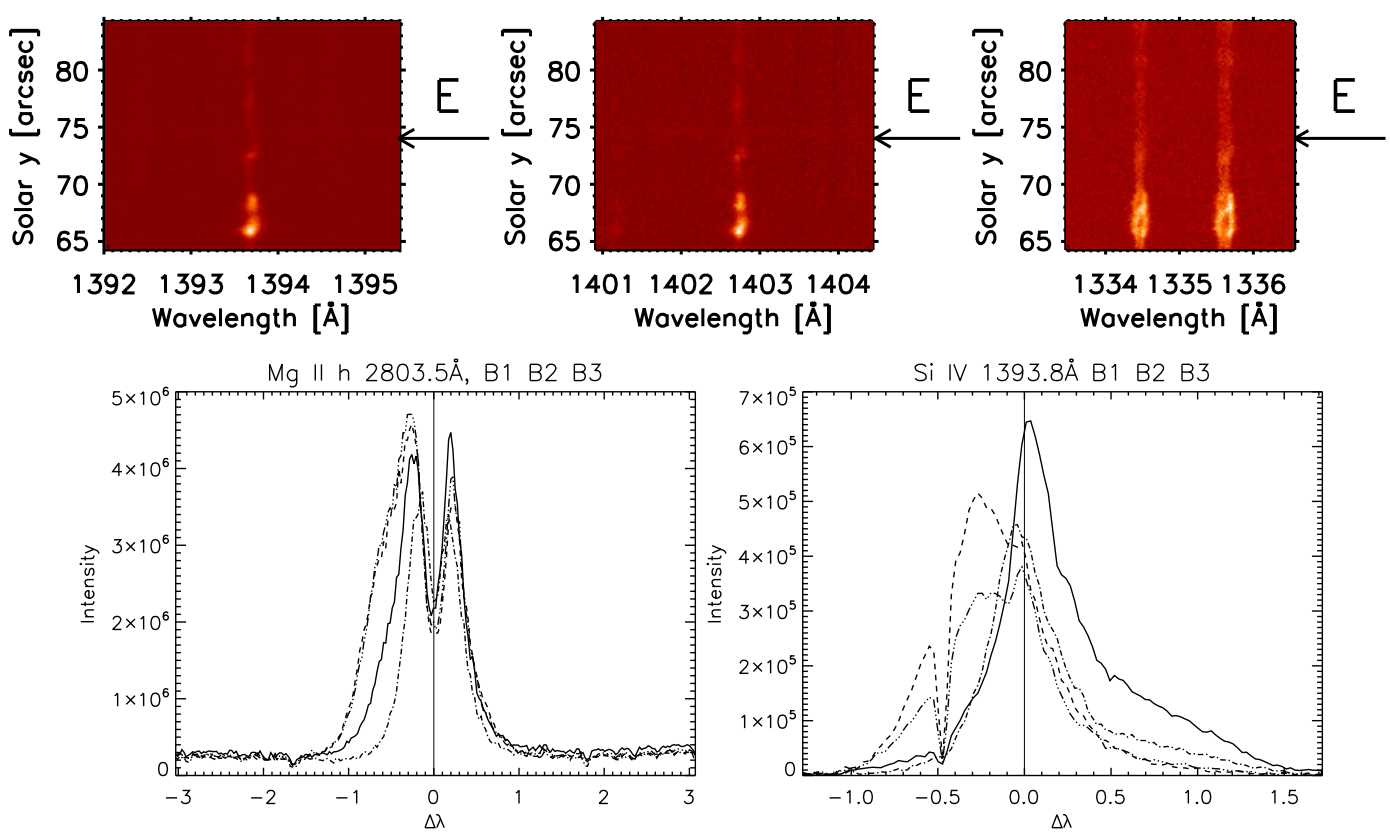

Fig. 6. Four top rows: spectra of Si IV $1394 \AA$, Si IV $1403 \AA$, and C II $1336 \AA$ Af the five selected A to E CBs. Bottom left and right panels: example of multi structures in CBs along the slit: Mg II h and Si IV $1394 \AA$ respectively profiles in the CB B: B1 identical to B for $y=59$ arcsec (pixel = 374) (solid line), B2 for $y=59-0.33$ arcsec (pixel = 372) (dashed line), B3 for $y=59+0.5 \operatorname{arcsec}($ pixel $=377)($ dashed-dotted-dotted line), with profiles outside the bomb $(y=61.5 \operatorname{arcsec}(\mathrm{pixel}=389)$ - dashed-dotted line). The bright spectra with a large blue shift below B is not considered as belonging to CB B. The Si IV 1393.76 line blue wing is ragged by two absorption lines (Fe II at $1392.2 \AA$ ) and Ni II (1393.3 $\mathrm{A}$ ) for CBs A B, and D. Intensity units are in $\left(\mathrm{erg} \mathrm{s}^{-1} \mathrm{~cm}^{-2} \AA^{-1} \mathrm{sr}^{-1}\right)$, wavelengths unit is $\AA$. 
Table 2. Parameters of the observed Mg II line profiles.

\begin{tabular}{llllll}
\hline \hline & $\mathrm{A}$ & $\mathrm{B}$ & $\mathrm{C}$ & $\mathrm{D}$ & $\mathrm{E}$ \\
\hline Mg II h line centre intensity h3 $\left[\mathrm{erg} \mathrm{s}^{-1} \mathrm{~cm}^{-2} \AA^{-1} \mathrm{sr}^{-1}\right]\left(\times 10^{6}\right)$ & 1.02 & 2.08 & 1.40 & 0.42 & 0.42 \\
Mean peak intensity h2 [erg s $\left.\mathrm{cm}^{-2} \AA^{-1} \mathrm{sr}^{-1}\right]\left(\times 10^{6}\right)$ & 3.51 & 4.32 & 1.74 & 1.88 & 0.65 \\
Peaks separation $\Delta \lambda$ peaks $[\AA]$ & 0.53 & 0.46 & 0.46 & 1.07 & 0.33 \\
Integrated intensity $\int I_{\mathrm{abs}} \mathrm{d} \lambda\left[\mathrm{erg} \mathrm{s}^{-1} \mathrm{~cm}^{-2} \AA^{-1} \mathrm{sr}^{-1}\right]\left(\times 10^{6}\right)$ & 4.49 & 4.25 & 2.71 & 3.84 & 1.33 \\
Line centre contrast & 4.08 & 8.72 & 5.51 & 0.97 & 0.95 \\
Mean peaks contrast & 8.22 & 9.34 & 3.52 & 3.95 & 0.69 \\
Mean contrast at $\Delta \lambda=+/-3.5 \AA$ & 2.86 & 2.17 & 4.43 & 3.86 & 3.53 \\
Contrast in continuum at $2830.4 \AA$ & 0.08 & 0.16 & 0.60 & 0.42 & 0.39 \\
h2v/h2r ratio & 0.99 & 0.94 & 1.53 & 1.08 & 1.15 \\
Type of the brightening & 1 & 1 & $2 \mathrm{a}$ & $2 \mathrm{~b}$ & 3 \\
Emission in Si IV and CII lines & $\mathrm{Y}$ & $\mathrm{Y}$ & $\mathrm{N}$ & $\mathrm{Y}$ & $\mathrm{N}$ \\
\hline
\end{tabular}

Notes. The contrasts are computed by comparison with the quiet Sun intensity at the disk centre.

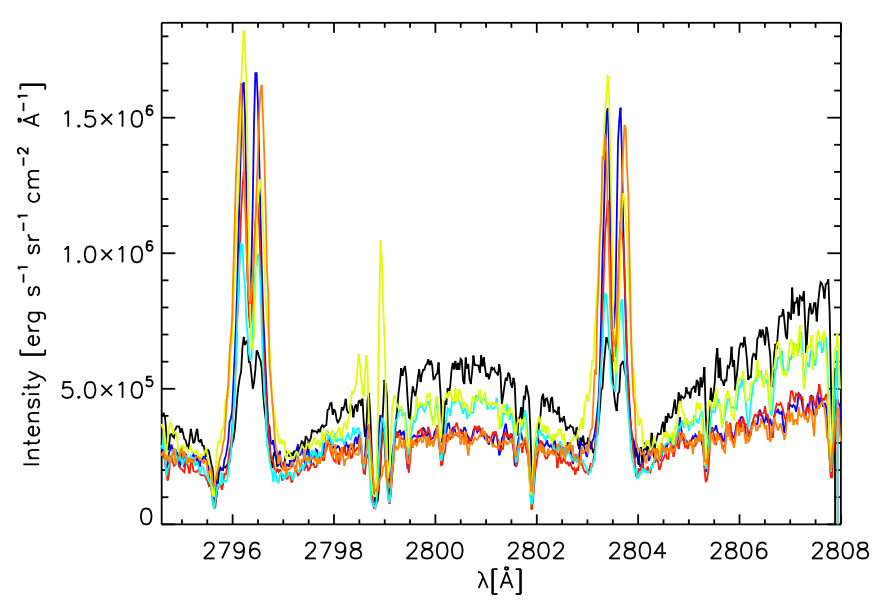

Fig. 7. Intensity profiles of $\mathrm{Mg}$ II lines for events that have high intensity in the wings and small intensity in the peaks. The selection was done randomly (see the small circles in Fig. 3). The correspondence is as follows: 5 - dark blue; 6 - light blue; 7 - red; 8 - orange; 9 - light green. The black line represents the $\mathrm{E}$ profile. All these profiles have a shape between $\mathrm{CB} C$ and $\mathrm{CB}$ E profiles.

example (Fig. 5). The two peaks $\mathrm{h} 2 \mathrm{v}$ and $\mathrm{h} 2 \mathrm{r}$ are very broad, separated, ragged and irregular, as was mentioned in Peter et al. (2014). The intensity of the peaks is comparable to the h2 intensity of the QS loc profiles (dashed lines in Fig. 5), but the line centre intensity h3 is lower than h3 of the QS ${ }_{\text {loc }}$, which is nearly equivalent to the $h 3$ value of the $\mathrm{QS}$. The wing emissions are several times higher than in the case of both the quiet Sun wing emissions (QS and $\mathrm{QS}_{\text {loc }}$ ). This CB (D) has a strong emission in the $\mathrm{Mg}$ II triplet between the $\mathrm{h}$ and $\mathrm{k} \mathrm{Mg}$ II lines around 2798.5 to $2799.5 \AA$ and strong emission in Si IV and CII lines with absorption of photospheric lines, which suggests the presence of a cloud of cool material along the line of sight at the location of the $\mathrm{CB}(\mathrm{D})$.

The (E) spectrum presents a completely different type of event. Bright emission is visible with small intensity differences between the peaks and the far wing emission. The $\mathrm{Mg}$ II h profile has peak intensity values $(\mathrm{h} 2 \mathrm{v}$ and $\mathrm{h} 2 \mathrm{r})$ that are only double the QS peak intensity. and more than 50\% even lower than the $\mathrm{QS}_{\mathrm{loc}}$. Peak emission is around one order lower than for the other CBs. CB (E) spectra in Si IV, CII lines, and Mg II triplet present no emission (Fig. 6). Type (C) to type (E) profiles are the more frequently observed $\mathrm{CB}$ profiles, see some other randomly chosen profiles (Fig. 7). They correspond to small CBs, shown by small circles in the spectroheliogram obtained in Mg II h-1 $\AA$ (Fig. 3, second row right panel).

\subsection{Classification of the Mg II compact brightenings}

After the detailed analysis of the Mg II profiles of the five CBs, we determined three different types of brightenings. We believe that later on, this will allow us to find some EBs among them. For each selected $\mathrm{CB}$, we determine the following parameters of the $\mathrm{Mg}$ II h profile: peak separation, line centre intensity, peak mean intensity, integrated intensity (with range: $-/+1.7 \AA$ ). These values will be useful in the next part on our analysis, for comparison with the synthetic profiles. All of these profile parameters are shown in Table 2. Three types have the following characteristics: (1) enhancement emission in peaks and close wings; (2) enhancement emission in peaks, close wings, and far wings; and (3) enhancement emission only in wings, close and far, and untouched in the peaks and line centre. We also note that the lower the emission is in the line, the higher the emission is in the wings and far wings. Our division is as follows: $\mathrm{CB}$ (A) and $\mathrm{CB}$ (B) brightenings represent the first type, $\mathrm{CBs}(\mathrm{C})$ and (D), the second type, the third type is the $\mathrm{CB}(\mathrm{E})$, which are very common features in our field of views in Mg II lines (Figs. 3 and 4). To characterize properties of profiles and test our classification a posteriori in three groups, we calculated the contrast values in comparison with the quiet Sun profile for each brightening for three wavelengths $(\mathrm{h} 3, \mathrm{~h} 2$ and $\delta(\lambda)+3.5 \AA)$. The contrast is defined as follows:

$C=\left(I_{\mathrm{CB}}-I_{\mathrm{QS}}\right) / I_{\mathrm{QS}}$,

where $I_{\mathrm{CB}}$ is the intensity of the compact brightening and $I_{\mathrm{QS}}$ is the intensity of the quiet Sun. These values of contrast are presented in Table 2.

Type 1 CBs have a strong contrast in the line centre and also in the mean peak, type $2 \mathrm{CBs}$ have a high contrast in the far wings, type 3 also has a high contrast in the far wings, but no contrast in the mean peak.

The five $\mathrm{CBs}$ that we selected have different $\mathrm{Mg}$ II profiles with different characteristics and different counterparts in Si IV, CII, and the Mg II triplet. This different behaviour helps us to clarify our selection of CBs in type 2. The Mg II triplet is particularly visible in the type 2 spectra of our selection $\mathrm{CB}(\mathrm{C})$ and (D) with enhancement of the emission to 

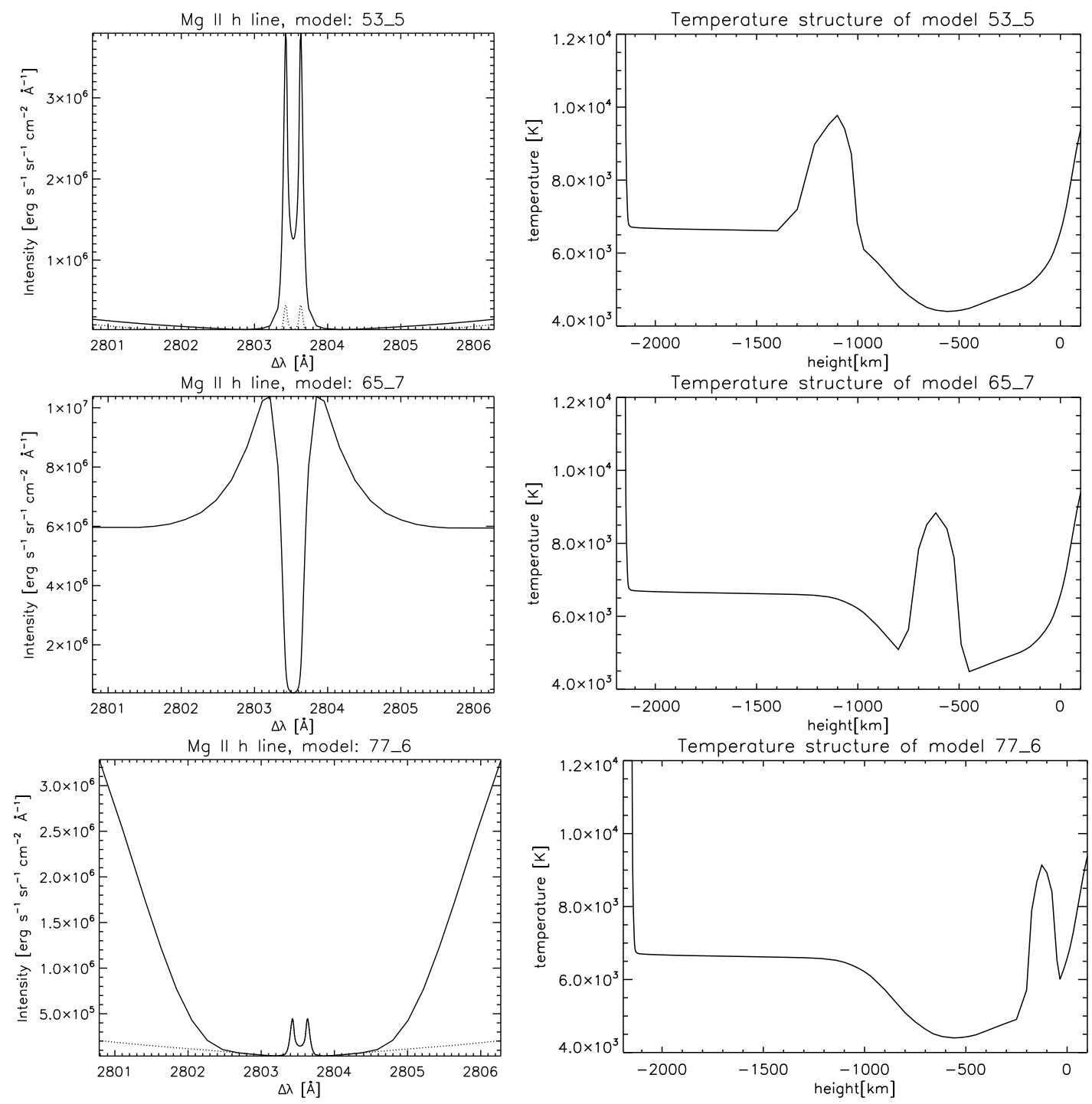

Fig. 8. Examples of synthetic profiles for different models: chromospheric 53-5, model of the hotspot in low chromosphere, close to the temperature minimum region 65-7 and photospheric model 77-6. For each model a different part of the profiles is enhanced.

$1-1.2 \times 10^{6} \mathrm{erg} \mathrm{s}^{-1} \mathrm{~cm}^{-2} \AA^{-1} \mathrm{sr}^{-1}$. This is why they belong to the same type. However, they present a strong difference in their signatures in the Si IV and CII lines. Therefore we classify CB (C) as type $2 \mathrm{a}$ and $\mathrm{CB}$ (D) as type $2 \mathrm{~b}$. Types 1 and $2 \mathrm{~b}$ commonly have strong signatures in hot temperature formation lines.

In Table 2, we add a line on the emission in Si IV line of each $\mathrm{CB}(\mathrm{Y} / \mathrm{N})$ and the $\mathrm{h} 2 \mathrm{v} / \mathrm{h} 2 \mathrm{r}$ ratio in $\mathrm{Mg}$ II $\mathrm{h}$ line, indicating the degree of asymmetry of the profile. The value of the $h 2 v / h 2 r$ ratio is close to 1 , indicating that the selected profiles are more or less symmetrical. Each category represents typical profiles that are observed.

The paper of Vissers et al. (2015) presents different IRIS profiles of CBs which overlay spatially and co-temporally $\mathrm{H} \alpha$ EBs observed with the SST. Some of our CBs present similar morphological characteristics and belong to this special category of CBs selected with a different criteria. The three CBs (A, B, and D) follow their criteria, being visible in Si IV. In fact they have either large emission in Si IV lines or wide profiles. Our CB (A) which is the bomb 3 of Peter et al. (2014) and our CB (B) have similar profile as EB2 in Vissers et al. (2015). They could be considered as $\mathrm{H} \alpha$ EBs and hot explosions. In relation to the distinction between the flaring active filament (FAF) and EB, the profile D (hot explosion 1 in Peter et al. 2014) is, maybe, an FAF as mentioned by Vissers et al. (2015) and not an EB in an early stage of life, but in a later phase when the AFS are making a strong canopy over the region.

The two other CBs of our selection, C (type 2a) and E (type 3), which are the more frequent in our selection (see Fig. 7) did not fit the Si IV criteria but we will see that the NLTE model that fits the $\mathrm{Mg}$ II profile of $\mathrm{CB}(\mathrm{E})$ reproduces well the $\mathrm{H} \alpha \mathrm{EB}$ that we have observed with THEMIS.

In summary we propose the following statistics concerning the $74 \mathrm{CBs}$ that we have detected in raster 1 and raster 2 in the emergence areas: $10 \%$ are similar to type 1 , only one CB has an Mg II profile like CB (D) (type 2b), $10 \%$ to type 3 and $80 \%$ are between type 2 a to type 3 . For this analysis, we looked at each CB Mg II line and Si IV line profiles.

\section{NLTE modelling of Ellerman bombs}

\subsection{Mg II line modelling}

To calculate the synthetic line profiles, we use a multilevel NLTE radiative-transfer modelling. The multilevel NLTE transfer problem is solved by using the multilevel accelerated lambda 

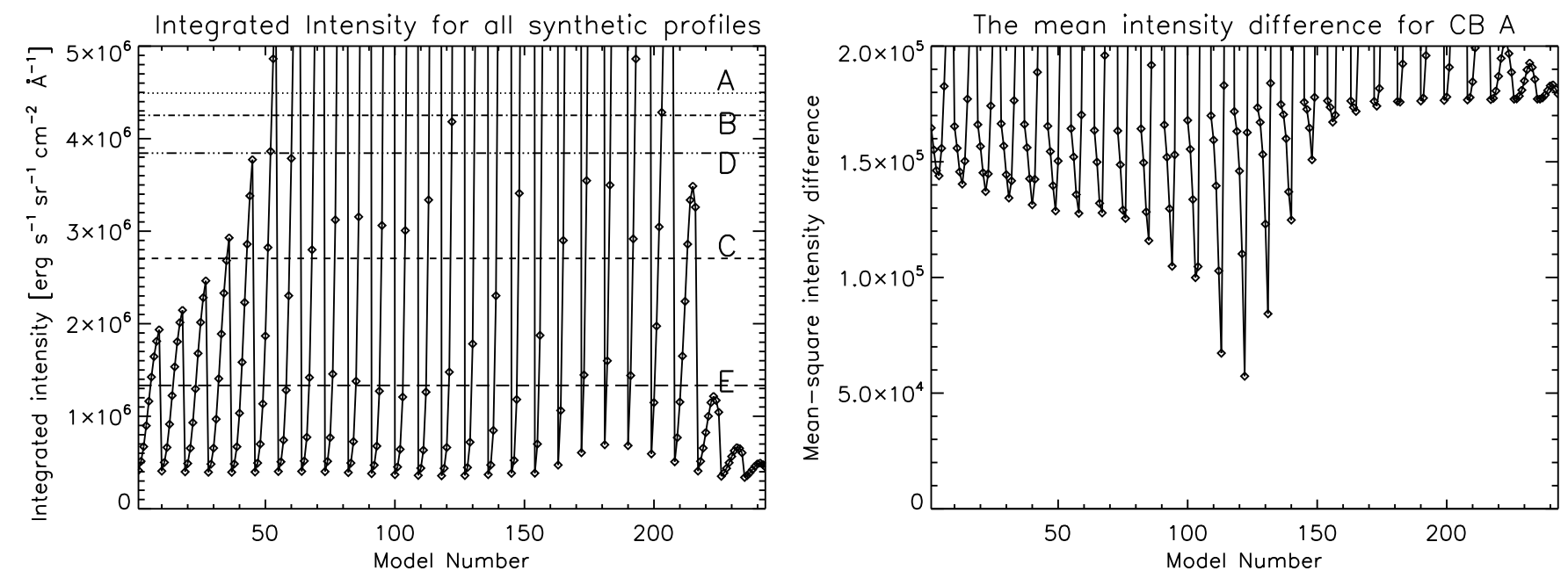

Fig. 9. Left panel: example of one profile parameter, such as the right integrated intensity obtained for all models. The horizontal lines on the diagram represent the values of the parameter for the observed profiles - dotted line for A brightening, dashed line for B, dashed dotted line $\mathrm{C}$, dashed-dotted-dotted-dotted for D and long dashed line for E. Right panel: the square root of the mean-square intensity difference between synthetic and observed profiles: one example of the A brightening. The best model for A is $N=120$.

iteration technique (MALI) of Rybicki \& Hummer (1991, 1992) and the linearization scheme to take into account the hydrogen ionization equilibrium (Heinzel 1995). This solves the radiative transfer and statistical equilibrium equations for our model of $\mathrm{Mg}$ II ion. In this study, we use a five-level plus continuum Mg II-Mg III model atom. Apart from the two strong $h$ and $k$ resonance lines, we also include three line transitions between the 3P and 3D states (Mg II triplet see Table 1). These subordinate lines appear just close to the $h$ and $k$ lines (2790.8, 2797.9, and $2798.0 \AA$ ). However, they are generally very weak and relatively far from $\mathrm{h}$ and $\mathrm{k}$ line peaks. Based on Milkey \& Mihalas (1974) (with the reference to Dumont 1967), who showed that these and other subordinate lines have a negligible effect on the source function of the resonance lines, we thus neglect other line transitions to $4 \mathrm{~S}$ state and to higher states. For our 1D modelling of EBs, this model of Mg II is fully sufficient. Finally, we solved the NLTE transfer problem and we synthesized the emergent hydrogen line profiles of EB. In the next step, we used the same model and computed electron densities to synthesize the $\mathrm{Mg}$ II $\mathrm{h}$ and $\mathrm{k}$ spectrum. Mg II $\mathrm{h}$ and $\mathrm{k}$ lines are treated with PRD, which is very important in the line wings. Atomic data and collisional rates provided by Shine \& Linsky (1974) and H. Uitenbroek (priv. comm.).

\subsection{Grid of models}

We used EB models based on a simple 1D plane-parallel, semiinfinite atmosphere. The construction of atmospheric model of EBs was discussed in Berlicki \& Heinzel (2014). These models were constructed by placing a local temperature increase (LTI, also known as a hot-spot) structure at a given altitude in the solar atmospheric model of the quiet Sun. The modelling performed in this study does not take into account the dynamics and will therefore not explain the asymmetry of some Mg II profiles. We used the semi-empirical model C7 developed by Avrett \& Loeser (2008). To find the best models that fit our observed profiles, we created a grid of models where the temperature structure is modified within the hotspot. Our model parameters are: the position/altitude of the hot spot and its width and peak temperature increase. Similar to Berlicki \& Heinzel (2014), our grid of models consisted of 243 models. We chose 27 different positions of the hotspot, every $40 \mathrm{~km}$, from $50 \mathrm{~km}$ above Sun surface $\left(\tau_{500}=1\right)$ to $1500 \mathrm{~km}$. For each position in the solar atmosphere, we have nine different models with different values of the temperature increase. The width of LTI does not change. Mass density of the hot spot is taken from a C7 model and we did not modify it. The sensitivity of the results to the density has been tested in Berlicki \& Heinzel (2014) and they concluded that the sensitivity was weak and the best fitting was obtained without changing density in CBs. In Fig. 8, we present some representative examples of the temperature profiles of some models from our grid. The names of models consist of two numbers - the first number from 52 to 78 (respectively 1500 and $50 \mathrm{~km}$ ) represents the position in the atmosphere and the second number represents the temperature increase of the hot spot, from 1 (for $1100 \mathrm{~K}$ ) to 9 (for $5500 \mathrm{~K}$ ). For each model, we computed the synthetic spectra of the $\mathrm{Mg} h$ and $\mathrm{k}$ lines.

It is well known that $\mathrm{Mg}$ II $\mathrm{h}$ and $\mathrm{k}$ lines are formed in the wide range of the chromosphere and upper photosphere and different parts of the line profiles come from different heights (Leenaarts et al. 2013a,b; Pereira et al. 2013). Therefore, magnesium $\mathrm{h}$ and $\mathrm{k}$ lines are good indicators of the atmospheric conditions. The greatest opacity is in the line centre and this part of the line is formed in the upper chromosphere. Further parts of the line profiles are formed deeper in the atmosphere. This relation is also clear in our examples (Fig. 8). For LTI placed high in the atmosphere, we obtain strong narrow peaks and a small emission in the wings. The deeper we move, the more the emission is enhanced and moves further away from the line centre and the peaks get wider, because of combined emission in peaks and close wings. For hotspots located around the temperature minimum, the emission in far wings became much stronger but the line peaks are still enhanced. For hotspots located even deeper in the photosphere, the emission is strongly enhanced only in the far wings, but the line centre intensity decreases and become close to the quiet Sun intensity of both Mg II lines.

\section{Comparison of the observed and synthetic spectra}

The grid of models contains a great number of hotspot models $(N=243)$. Our goal is to find the best model of each selected 

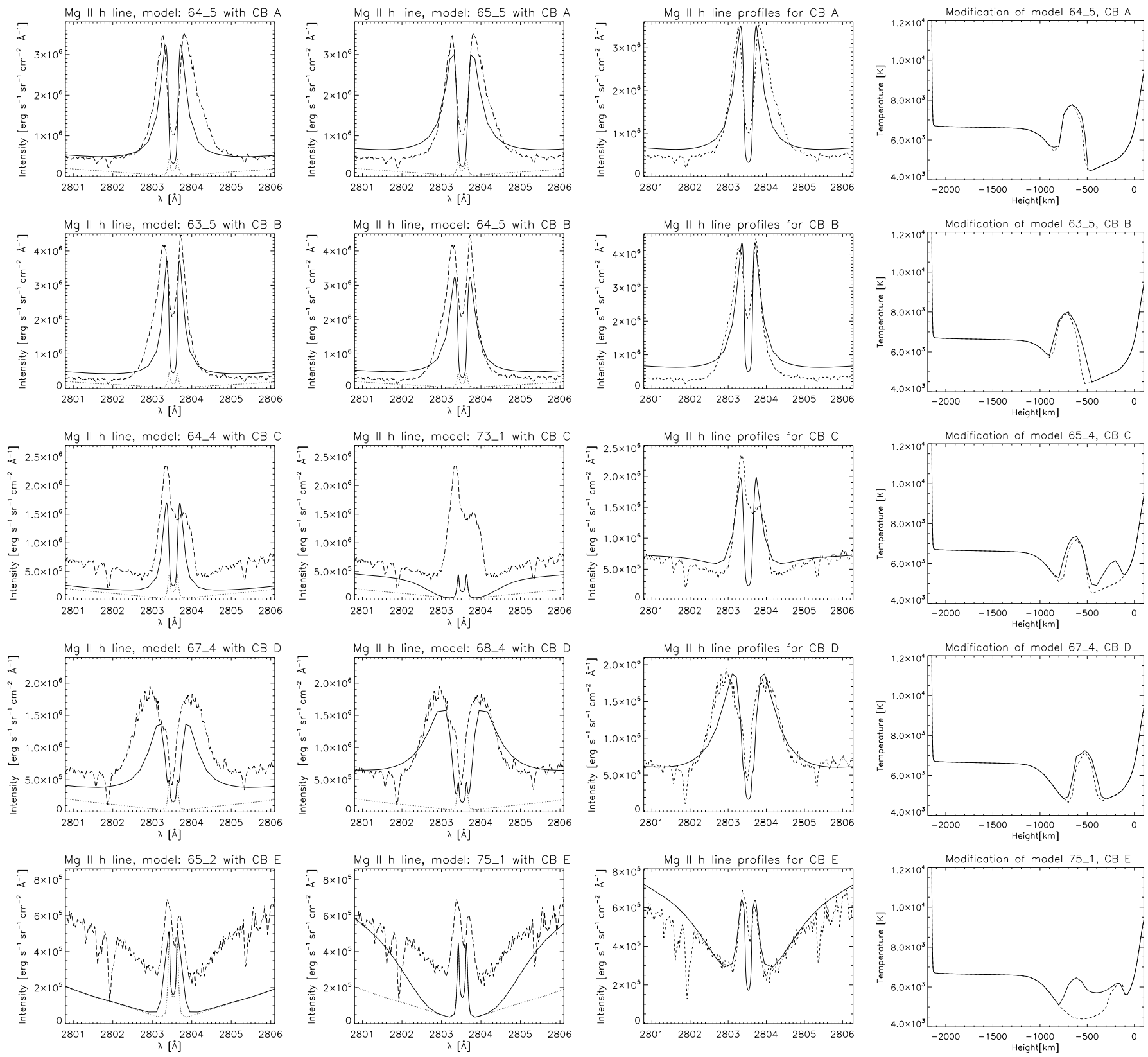

Fig. 10. Plots with the results of modelling. The first two left columns show some synthetic profiles (solid line) compared with observational profiles (dashed line) for best models from the grid. Some of the profiles are chosen from method based on profiles parameters, some of them have additional profile chosen after visual verification (63-5 for B, 73-1 for C, 68-4 for D, 75-1 for E). The third column presents the synthetic profiles (solid line) obtained from modified models compared with observational profiles (dashed line). In the last, right column, we present the temperature structure of the best models plotted with a black solid line. The dashed line represents models before modification. In this figure, each row corresponds to other compact brightening from A to E, respectively.

bright $\mathrm{Mg}$ II bright points from these $N$ models. To compare synthetic and observed line profiles, we take into account four different parameters of each profile, both for the synthetic and the observed: peak separation, intensity of the peak (in the case of observed spectra we took average intensity from two peaks), intensity of the line centre and integrated intensity (Table 2). Integrated intensity was computed in the range from $-1.7 \AA$ to $+1.7 \AA$. In Fig. 9 (left panel), we show for each model the value of the integrated intensity parameter as an example. Five different horizontal lines in the plots represent the values obtained from observations. If the line passes through the value of this parameter for some models (diamond points), it means that this value fits with the observation. At first glance, many of the models fit the observations reasonably well. The method of looking for the best model through parameter fitting is not fully effective. The problem is that the model that is the best according to one parameter, is not good for another one. Our code does not include plasma flows, which may be important in the Mg II line formation. In the observed $\mathrm{CBs}$, many $\mathrm{Mg}$ II line profiles have asymmetric peaks, however, our selection was restricted to symmetric profiles where the asymmetry of peaks are not significant, suggesting that the velocity of the emitting plasma is low. This weak flow should not affect all four parameters, which were used for model determination, but mainly the separation and the intensity of the peaks. Peak separation or line centre intensity do not seem to be a proper parameter to determine the best model. From 
the whole set of best models, we can derive some kind of distribution of models for each parameter, which can tell us about the approximate region of the atmosphere, where the CFs are forming: are they in the photosphere or rather in chromosphere? If we knew that, we could narrow down our search for the best models.

Our analysis showed that the most suitable parameter for the model determination is the line-integrated intensity. For each given line, emitted under specific conditions, the shape of the profile can be different because of the Dopplershifts, but the total emission, represented by integrated intensity, should be the same. We also computed the mean intensity difference between all synthetic profiles and each observed profile (in the wavelength range: $2800.8-2806.5 \AA$ ) in the following way:

$\Delta I=\frac{\sqrt{\sum_{\beta=1}^{n}\left(I_{\mathrm{CB}}(\lambda)-I_{\mathrm{SP}}(\lambda)\right)^{2}}}{n}$,

where $I_{\mathrm{CB}}$ is the intensity of the compact brightening, $I_{\mathrm{SP}}$ is the intensity of synthetic profile, $n$ is the number of wavelength points across the line profile. Figure 9 (right panel) presents one example diagram of the mean-square intensity difference between all synthetic profiles and the observed profile for compact brightening $A$. The best models have the lowest values of meansquare intensity difference, e.g. for $A$ it is $N=120$.

\section{Models of bright points obtained from Mg II line profiles}

All the presented methods of fitting the theoretical and observed line profiles only give us an approximate model. General properties of hotspots (position, temperature, and width) are presented in Table 3. If we consider each fitting parameter separately, we get some indication of the hot spot parameters: the approximate position in the atmosphere and the temperature increase. Each $N$ model is defined by two numbers, one characterizing the position and one the temperature. The model for which there is a convergence towards these two parameters was chosen as the best model, after a visual inspection. Some models of CB were not found by our methods and we had to apply a new type of model with two hotspots.

In Fig. 10, we present the results of the modelling using the method described in the previous section. In some cases a modification of the models is required, because of the adopted discretization of temperature and the unique value of the width for each peak. It can happen that the modelled temperature of the hotspot or the width of the line peak has to be between two grid values to obtain the best agreement with observations. We noticed in Fig. 10 that for A brightening the fitting is not perfect, the peaks are too small, but we can change it by raising the temperature of the peak slightly in the two chosen models (64-5, 65-5). The profile calculated with the model $64-5$ has too small an emission in the wings, and too narrow peaks. In the case of 65-5 model, we have the opposite situation, despite this model being different only by its position in the atmosphere, by around $40 \mathrm{~km}$. The hotspot of this model is at a height of around $630 \mathrm{~km}$ above the Sun's surface, in the low chromosphere. We can assume that if we also modify the width of the temperature peak, we can link these two models as one.

In the case of fitting the B brightening, we have a similar situation, the best model is also (64-5), but it required higher temperature peaks. Additionally, we chose model 63-5; it has smaller emission in the wings and higher and narrower peaks. By expanding and increasing the temperature of LTI we obtain broader peaks (Fig. 10). The problem with the line centre and intensity in wings is similar to the A brightening case. In this profile, we have a red asymmetry of the peaks, which indicates the presence of flows. B brightening represents a dynamic event and the profile presents a red asymmetry, as can be seen in the spectrum (for cut $y=374$, for other cuts profiles is changing diametrically). For now, we do not focus on the dynamics of the events, but this should be taking into account in future analysis of this phenomena, especially sunce it can be a signature of explosions, which have strong influence on the profile and on the model of the phenomena itself.

Also D brightening has quite a good fitting and the best models for this event have peaks placed deeper in the atmosphere and have a lower temperature LTI. Model 68-4 is chosen by our methods and model 67-4 is an additional model, chosen by visualization. The profile obtained from model 68-4 for D brightening does not correctly fit the wing and peak intensity, even after modifications (Fig. 10). Therefore, we selected the other model, 67-4, in which we change the width of the LTI and the increase the temperature. The fitting is better for the red peak. Nevertheless the observed profiles cannot be completely recovered because the observed line centre is blueshifted (owing to flows), and the peaks are asymmetrical and jagged, which again indicates the presence of dynamic properties of plasma.

We had a bigger problem with $\mathrm{C}$ and $\mathrm{E}$ brightening. Examples of $\mathrm{C}$ and $\mathrm{E}$ events reveal that the temperature structure of LTI is more complex. Increased intensity, both in the peaks and in the wings, indicate a very broad region of the atmosphere where the temperature was increased. Even a small enhancement in the peaks of E profile, needs some LTI higher in the atmosphere. The very first step in finding the best model is a simple combination of these two best models.

$\mathrm{C}$ and $\mathrm{E}$ brightenings require a different treatment if we want to obtain a better fitting. In both, $C$ and $E$ cases, we take two models for which some parts of the synthetic profiles were in agreement with the observational profiles and we combined the profiles together. Then we modify these linked models, to obtain the best possible fitting, by rising or decreasing the temperature in this region of the atmosphere, where particular parts of the profiles are formed. For C brightening, we took models $64-4$ and 73-1. In position 73, in our model of the atmosphere the magnesium wing starts to rise, which is necessary if we want to get a strong emission in wings like in the observed profile. These two combined models are presented in Fig. 10 (middle right panel). We expanded and increased the first LTI at $650 \mathrm{~km}$ and the second LTI at $200 \mathrm{~km}$. The asymmetry of the C profile suggests the presence of velocities in the CB's atmosphere and possibly in the overlying fibrils (see the next section). This asymmetry was problematic in the modelling and we decided to chose the mean intensity of peaks in the observed profile. A similar situation arises in the case of $\mathrm{E}$ brightening. The two best models 65-2 and 75-1 were combined and modified (bottom right panel in Fig. 10). Because of the small intensity in this event, the LTIs of the two hotspot is small and does not have such a deep minimum temperature between them. First we increased LTI at $650 \mathrm{~km}$ and raised the temperature between the two temperature peaks. In this case the fitting is very good.

We also estimated the quality of the fitting of the profiles in percents for the integrated intensity in the following way:

$F i t=\left(1-\left|\frac{I_{\text {intobs }}-I_{\text {intsyn }}}{I_{\text {intobs }}}\right|\right) \cdot 100 \%$,

where $I_{\text {int }}$ means the value of integrated intensity for synthetic and observational profiles. As we can see in Table 3, for all 
Table 3. Parameters hotspot.

\begin{tabular}{llllll}
\hline \hline & A & B & C & D & E \\
\hline Hot spot range $[\mathrm{km}]$ & $450-850$ & $450-900$ & $100-800$ & $300-750$ & $75-800$ \\
Temperature peak $[\mathrm{km}]$ & 660 & 700 & 615 and 200 & 525 & 660 and 175 \\
Temperature at peak position $[\mathrm{K}]$ & 7750 & 8000 & 7350 and 6150 & 7250 & 6350 and 6200 \\
Temperature peak $(\Delta T)[\mathrm{K}]$ & 3250 & 3350 & 2900 and 1150 & 2850 & 1850 and 1100 \\
Fit of profiles $[\%]$ & $91.9 \%$ & $96.5 \%$ & $99.4 \%$ & $93.7 \%$ & $93.8 \%$ \\
\hline
\end{tabular}

brightenings, the fitting parameter is higher than $90 \%$. The best result we got is for $\mathrm{C}$ brightening, the worst for the $\mathrm{A}$ event, which was easily predictable by taking into account the different emissions in the wings and the narrow wings. As mentioned in the previous section, integrated intensity represents the energy emitted within the line and it is not dependent, to some extent, e.g. on the plasma flows. Since we are not analysing the dynamics of $\mathrm{CBs}$, we decided to used this parameter as a good representative of the physical conditions in the CBs. In the case of $\mathrm{C}$ and $\mathrm{E}$ events, we put two values of position, the first refers to the higher LTI, the second to the lowest. The same rule applies for temperature. Range of heights of the hotspot covers two LTIs, from the beginning of first to the end of the second. We notice some relationship between the shape of the profile and its position in the atmosphere, as mentioned above. A higher emission in the line peaks indicates a higher position of the hotspot in the atmosphere. Moreover, the more enhanced emission in the wings suggests the deeper position of the temperature increase. We can also claim that, even if the brightenings are formed at different positions, these heights are different only by about $400 \mathrm{~km}$, which for the Sun-scale is not so great. It is clear that, generally, our brightenings are formed in the lower chromosphere to the upper photosphere, close to the temperature minimum region, which is in good agreement with the results of previous work (Berlicki \& Heinzel 2014).

\section{An effect of the overlying canopy}

Arch filament systems are commonly overlying emerging flux regions and form a chromospheric canopy (Kitai 1983; Dara et al. 1997; Rutten et al. 2013). This canopy may have an important effect on the intensities in the cores of studied lines in $\mathrm{CB} / \mathrm{EBs}$. The canopy consists of many fine-structure elements that may extend to altitudes as high as a few thousands $\mathrm{km}$ and, indeed, may obscure the EB (see also recent study of Hong et al. 2014 based on classical cloud model). However, as already demonstrated in Heinzel \& Schmieder (1994, see also references therein), these elements usually called mottles or fibrils may posses a range of temperatures and gas pressures that lead to the $\mathrm{H} \alpha$ line core contrast varying from absorption to emission. This is why Heinzel \& Schmieder (1994) called them "black and white mottles". In case of optically-thick lines like $\mathrm{Mg}$ II $\mathrm{h}$ and $\mathrm{k}$, the line centre will thus exhibit the radiation of a mottle, while the peaks may result from a superposition of an attenuated radiation of $\mathrm{EB}$ and emission of the mottle itself. In the line wings we should see the EB's and CB's radiation unaffected by the canopy. In Fig. 3, we clearly see thin fibrils which may obscure some brightenings. Especially, CB A, C, and D seem to be overlaid by darker or brighter threads that are well visible in the $\mathrm{Mg}$ II h line centre.

Simple consideration of the formal solution of the transfer equation shows that the spectral line profile emitted by $\mathrm{CBs}$

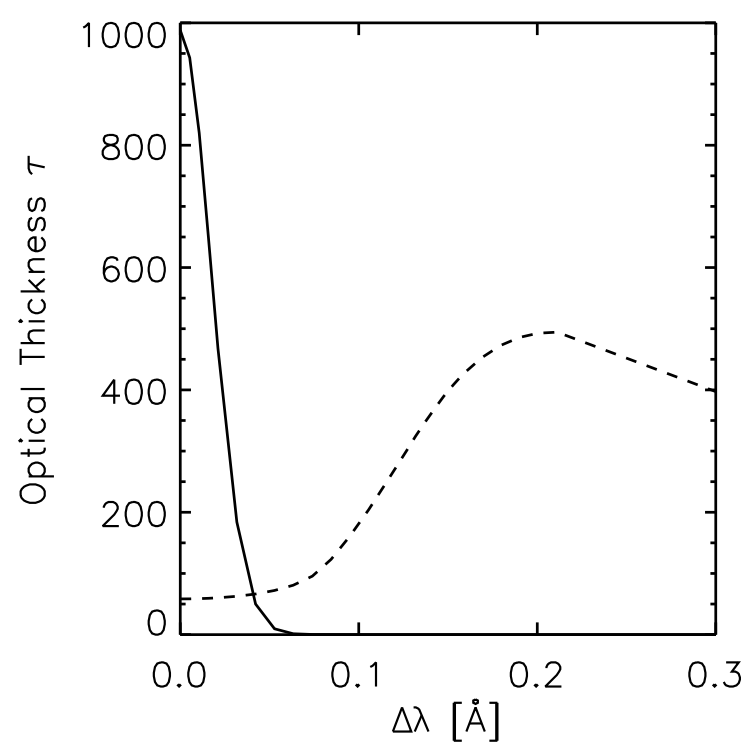

Fig. 11. Theoretical optical thickness $\tau$ of fibril within $\mathrm{Mg}$ II $\mathrm{h}$ line (solid line) calculated with 2D codes using our fibril model, and the theoretical $\mathrm{Mg}$ II $\mathrm{h}$ line profile for the CB C modified model 65-4 (dashed line) calculated using our NLTE chromospheric codes. The fibril model parameters are $D=2000 \mathrm{~km}, T=10000 \mathrm{~K}$ (isothermal), $p=2 \mathrm{dyn} \mathrm{cm}^{-2}$ (isobaric). The $x$ scale is given in $\AA$ ( $0.1 \AA$ corresponds to $\left.4.5 \mathrm{~km} \mathrm{~s}^{-1}\right)$.

should be a composite of the fibril and CBs profiles:

$I(\lambda)=I_{\mathrm{CB}}(\lambda) \exp [-\tau(\lambda)]+I_{F}(\lambda)$,

where $I_{\mathrm{CB}}$ is the emergent intensity of the $\mathrm{CB}$ modelled in this paper as a temperature perturbation of the $\mathrm{C} 7$ model, $I_{\mathrm{F}}$ is the intensity of the fibril emergent from its top surface, and $\tau$ is the wavelength-dependent optical thickness of the fibril. We note that this formula represents a generalized cloud model with the variable line source function (classical cloud model of Beckers 1964 assumes a constant source function).

To estimate quantitatively the possible effects of the canopy, we performed preliminary NLTE modelling of fibrils that horizontally overlie the CBs. Fibrils are modelled as flux tubes with the cross-section much narrower compared to their length and for this geometry we apply a $2 \mathrm{D}$ radiative-transfer model. We considered a 2D rectangular box with af $2000 \mathrm{~km}$ width placed horizontally above the solar surface at an altitude of $5000 \mathrm{~km}$ (we note that the horizontal extension is infinite in the model). This structure is isothermal and isobaric and the box is illuminated by the solar disk radiation. We assumed the temperature of $1 \times 10^{4} \mathrm{~K}$ and the pressure of $2 \mathrm{dyn} / \mathrm{cm}^{2}$. These values are based on previous studies of Heinzel et al. (1992), where the parameters of dark loops visible against the solar disk were discussed. At this altitude, the bottom of the box receives almost undiluted disk radiation, while both vertical sides are illuminated with the dilution factor of around one half. The top surface is not illuminated 
because the corona does not emit in Mg II lines. The thickness and the height of fibril was assumed according to the typical values obtained from observations. We adapted the 2D NLTE code described in Heinzel \& Anzer (2001) and Heinzel et al. (2015) to compute the intensity of the fibril itself that was emergent from the top surface (e.g. as seen against the disk), and we also obtained the total optical thickness of the fibril over the line profile.

In Fig. 11 we see that the $\mathrm{Mg}$ II h line-centre optical thickness is enormous in the fibril and thus what we observe - provided that the CBs is obscured by the canopy - is the fibril radiation $I_{\mathrm{F}}$. However, it is very interesting to observe that at the location of the $\mathrm{Mg}$ II line peaks around $0.2 \AA$, the optical thickness of the fibril is already very small $(\tau \approx 0.01)$, and thus the observed peaks indeed likely represent the radiation $I_{\mathrm{CB}}$ of the CBs.

We note that by introducing flows in the fibril (typically of the order 10-20 $\mathrm{km} \mathrm{s}^{-1}$ (see Heinzel \& Schmieder 1994), we could account for the observed asymmetry of the peaks by the Doppler shift of the line-core absorption.

Our very preliminary tests showed that introducing a canopy fibril can explain the observed line centre intensities which depend on the fibril parameters. But the Mg II line peaks already represent the emission from the perturbed region of the quiet Sun atmosphere, i.e. the EBs or CBs. This conclusion is important in the case of the CB C profile: by introducing the overlying fibrils, this enbales a better fitting of the $\mathrm{Mg}$ II h line centre intensity. Since the observed line centre intensity of $\mathrm{CB} C$ is higher than in our theoretical profile, in this case the fibril should be in emission with respect to the surrounding chromosphere. In our next paper, we plan to perform systematic modelling of these types of fibrils, also including the flows, and compare the theoretical composite $\mathrm{Mg}$ II line profiles with the observed line spectra.

\section{Discussion and conclusion}

We have revisited the IRIS observations of the flux emergence of September 24, 2013 observed at 11:44 UT (Peter et al. 2014). We have also analysed the next raster starting at 15:39 UT to have a better statistics. The aim of the paper was to detect possible Ellerman bombs (EBs) in these two fields of view by using the $\mathrm{Mg}$ II spectra. EBs are well recognized in spectra of chromospheric lines ( $\mathrm{H} \alpha$ and $\mathrm{Ca}$ II) by their so-called moustaches, with enhanced emission in the wings of the lines up to $10 \AA$. By way of an analogy, we investigated the wing intensities of $\mathrm{Mg}$ II lines, particularly $\mathrm{Mg}$ II $\mathrm{h}$ for practical reasons: the red wing of $\mathrm{Mg}$ II $\mathrm{h}$ is recorded along $4 \AA$ and doesn't overlap with the Mg II k line red wing. Using spectroheliograms reconstructed from the IRIS Mg II spectra, we identified 74 compact bright points (CBs) (38 and 36 respectively in each raster). They are mainly detected in the wings of the Mg II lines (at $1 \AA$ and $3.5 \AA$ ). Only eight of them are also observed in the Mg II h2 peaks (at $0.23 \AA$ ). We classified the CBs by their contrasts in the peaks, in the wings, far wings, and quasi-continuum at $2830.4 \AA$ in three categories (Table 2). The first type (the eight bright points) concerns bright points that have strong peak intensity and large contrast in the wings. These bright points belong to those that are clearly visible in Si IV lines and called "hot explosions" in Peter et al. (2014). We selected two of them and considered the more symmetrical profiles in the middle of each bomb (CBs A and B). The second type (CBs $\mathrm{C}$ and $\mathrm{D}$ ) concerns the $\mathrm{CBs}$ that have large integrated intensities because of the large width of their profiles and not because of their peak intensities. They both have a strong signature in the $\mathrm{Mg}$ II triplet line that has a chromospheric formation temperature, just below the transition region temperature.

The third type of CBs (E), is an extreme case of type 2a in the spectra. The profile has very a low peak intensity and high intensity in the far wing and continuum. They have no signatures in Si IV and CII lines like CB (C).

Our main goal was to compute the formation height of these five examples of CBs (A to E) visible in $\mathrm{Mg}$ II lines. Therefore we applied the NLTE radiative transfer code used by Berlicki \& Heinzel (2014) for $\mathrm{H} \alpha$ and Ca II lines to get synthetic $\mathrm{Mg}$ II line profiles to compare with the observations. The $1 \mathrm{D}$ atmosphere temperature profile is implemented with a hot temperature spot or local temperature increase (LTI) defined by a few parameters, such as the position, the width, and the amplitude. The density value is fixed because the model is relatively insensitive to density variations (Berlicki \& Heinzel 2014). We computed a grid of models and a grid of the corresponding Mg II and $\mathrm{H} \alpha$ synthetic profiles.

We have used an automatic detection method that is based on the parameters determined for each type of $\mathrm{Mg}$ II $\mathrm{h}$ profiles and then refined the search by visual inspection to fit the observed profiles with synthetic profiles. Five CB models were computed, one for each $\mathrm{CB}$ (A to $\mathrm{E}$ ) with one or two hotspots in the temperature profile. The hotspots are located in the photosphere between $75 \mathrm{~km}$ to $300 \mathrm{~km}$ and, for some of them, reached the chromosphere up to $900 \mathrm{~km}$. According to our statistics, $80 \%$ of the CBs have a type between $\mathrm{C}$ and $\mathrm{E}$, the profiles are relatively well fitted with an extended hotspot with two peaks at a distance of $700 \mathrm{~km}$ reaching two different temperatures around $6200 \mathrm{~K}$ and about $7350 \mathrm{~K}$. The increase of temperature reaches 3350 degrees. However the centre of the line $h_{3}$ is generally higher than the $h_{3}$ of the QS. This can be the effect of the overlying fibrils. To test this effect, we have used a 2D NLTE static model of fibrils. We show that this model can increase $h_{3}$ in the observed proportion, but does not affect the $\mathrm{h}_{2}$ peaks of the $\mathrm{Mg}$ II $\mathrm{h}$ line profile.

The question about the existence of EBs in $\mathrm{Mg}$ II $\mathrm{h}$ and $\mathrm{k}$ lines is important. EBs are mainly defined by the $\mathrm{H} \alpha$ line profiles. To be sure about the properties of EBs in Mg II h and $\mathrm{k}$ lines we need simultaneous observations in $\mathrm{H} \alpha$, as in the papers of Vissers et al. (2015) and Kim et al. (2015). Our observation of AR11850 in $\mathrm{H} \alpha$ is from the same day, but not simultaneous. Therefore they cannot be used directly. We attempt the comparison with our theoretical models and derived the synthetic profiles of $\mathrm{H} \alpha$ for the five $\mathrm{CB}$ models $\mathrm{A}$ to $\mathrm{E}$ (colour profiles in Fig. 2). The $\mathrm{H} \alpha$ synthetic profiles are consistent either with $\mathrm{H} \alpha$ THEMIS profiles (CB E) (black profile in Fig. 2) and/or with those obtained with the SST/CRISP and the NST (Vissers et al. 2015; Kim et al. 2015). The wings of the $\mathrm{H} \alpha \mathrm{C}$ profile have enhanced intensities up to $25 \%$ in $\mathrm{H} \alpha+/-2 \AA$ similar to the EBs of the NST (see the Fig. 5 in Kim et al. 2015) and up to $50 \%$ at $\mathrm{H} \alpha-1.25 \AA$ as for EB1 (see Fig. 4 in Vissers et al. 2015).

A discussion on the type 1 and type $2 b$ of CBs that have signatures in Si IV and CII lines show that these CBs are comparable to the hot explosions observed in Peter et al. (2014; their bombs 3 and 4 correspond to $\mathrm{CBs} \mathrm{A}$ and $\mathrm{B}$ ). Their corresponding $\mathrm{H} \alpha$ synthetic profiles obtained with these two $\mathrm{CB}$ types using our CB models (models A, B, and C) correspond to the profiles of EB1 and EB2 observed by Vissers et al. (2015; see their Figs. 4 and 8 top left panel) who found associated $\mathrm{H} \alpha \mathrm{EBs}$ in the field of view of SST. The CB D profile is closer to the profile FAF1 of Vissers et al. (2015; see their Fig. 13, top left panel) and can correspond to EBs overlaid by AFS. The test of the 2D model of 
the fibril was not done on this profile whose intensity and shape are far from being a classical Mg II profile. This should be done in a more extensive study on the influence of overlying fibrils.

To conclude, the three types of $\mathrm{Mg}$ II profiles could correspond to EBs. The reconnection seems to occur in the low atmosphere and could correspond to some plasmoid instability (Ni et al. 2015). The fast evolution of the bidirectional flows observed in hot lines (Si IV and C II) correlated with a weak EB would correspond more to a fast reconnection in the upper atmosphere (Kim et al. 2015). The model of reconnection in the corona of Archontis \& Hansteen (2014) in this way is a promising simulation to explain the type of explosive events observed in Si IV lines, but their model cannot really explain the formation of simultaneous $\mathrm{H} \alpha \mathrm{EBs}$, which requires a reconnection in the photosphere. In the paper of Dudík et al. (2014), there is interesting information about the temperature of the formation of Si IV lines in a non-Maxwellian distribution of electrons. With a $\kappa$ distribution, the temperature of the formation of Si IV can be just a chromospheric temperature and the intensity of the O IV line would be reduced, which could also explain the absence of signature of O IV in the hot explosions of Peter et al. (2014). An alternative to this scenario has been proposed by Judge (2015), taking into consideration the existence of Alfvénic turbulence in the plasma. The mechanisms of the formation of EBs are likely even more complex than was thought. This topic is very open. A further diagnostic of the physical properties from the line profile should help us determine the conditions in the atmosphere (Leenaarts et al. 2013b).

Acknowledgements. We would like to thank the anonymous referee who helped us to bring the manuscript to the acceptation stage owing to his/her helpful comments. We want to thank Bernard Gelly, the director of THEMIS and his colleagues for the observations with THEMIS/MTR instrument, to thank the observers of the Meudon solar tower (Regis Lecocguen and Daniel Crussaire) for the MSDP data. We thank Nicole Mein for the MSDP data reduction and the coalignement of the MSDP images with the IRIS images. We thank Liu Tian who provided us with the additional IRIS software that he had personally developed and Véronique Bommier for the THEMIS interface software, which we used for getting the $\mathrm{H} \alpha$ line profiles. This work is partially supported by the European Community Seventh Framework Programme (FP7/2007-2013) under grant agreement No. 606862 (F-CHROMA), by the grant of Polish Ministry of Sciences and Higher Education No. 3243/7.PR/14/2015/2 (2014-2016), by the grant No. 16-18495S of the Grant Agency of the Czech Republic and by the institutional grant RVO 67985815. IRIS is a NASA small explorer mission developed and operated by LMSAL with mission operations executed at NASA Ames Research Center and major contributions to downlink communications funded by ESA and the Norwegian Space Centre. The National Center for Atmospheric Research is sponsored by the National Science Foundation. We thank ISSI (Bern) for the workshop on "UV burst" managed by P. Young, which helped us to clarify our studies on the relationship between UB bursts and Ellerman bombs.

\section{References}

Archontis, V., \& Hansteen, V. 2014, ApJ, 788, L2

Archontis, V., \& Hood, A. W. 2009, A\&A, 508, 1469

Avrett, E. H., \& Loeser, R. 2008, ApJS, 175, 229

Beckers, J. M. 1964, Ph.D. Thesis, Sacramento Peak Observatory, Air Force Cambridge Research Laboratories, Mass., USA

Berlicki, A., \& Heinzel, P. 2014, A\&A, 567, A110

Berlicki, A., Heinzel, P., Schmieder, B., Mein, P., \& Mein, N. 2005, A\&A, 430, 679
Bernasconi, P. N., Rust, D. M., Georgoulis, M. K., \& Labonte, B. J. 2002, Sol. Phys., 209, 119

Cheung, M. C. M., Schüssler, M., Tarbell, T. D., \& Title, A. M. 2008, ApJ, 687, 1373

Dara, H. C., Alissandrakis, C. E., Zachariadis, T. G., \& Georgakilas, A. A. 1997, A\&A, 322, 653

David, K.-H. 1961, Z. Astrophys., 53, 37

De Pontieu, B., Title, A. M., Lemen, J. R., et al. 2014, Sol. Phys., 289, 2733

Dudík, J., Del Zanna, G., Dzif̌cáková, E., Mason, H. E., \& Golub, L. 2014, ApJ, 780, L12

Dumont, S. 1967, Ann. Astrophys., 30, 861

Ellerman, F. 1917, ApJ, 46, 298

Fang, C., Tang, Y. H., Xu, Z., Ding, M. D., \& Chen, P. F. 2006, ApJ, 643, 1325 Georgoulis, M. K., Rust, D. M., Bernasconi, P. N., \& Schmieder, B. 2002, ApJ, 575,506

Hashimoto, Y., Kitai, R., Ichimoto, K., et al. 2010, PASJ, 62, 879

Heinzel, P. 1995, A\&A, 299, 563

Heinzel, P., \& Anzer, U. 2001, A\&A, 375, 1082

Heinzel, P., \& Schmieder, B. 1994, A\&A, 282, 939

Heinzel, P., Schmieder, B., \& Mein, P. 1992, Sol. Phys., 139, 81

Heinzel, P., Schmieder, B., Mein, N., \& Gunár, S. 2015, ApJ, 800, L13

Herlender, M., \& Berlicki, A. 2011, Central European Astrophysical Bulletin, 35,181

Hong, J., Ding, M. D., Li, Y., Fang, C., \& Cao, W. 2014, ApJ, 792, 13

Isobe, H., Tripathi, D., \& Archontis, V. 2007, ApJ, 657, L53

Judge, P. G. 2015, ApJ, 808, 116

Kim, Y.-H., Yurchyshyn, V., Bong, S.-C., et al. 2015, ApJ, 810, 38

Kitai, R. 1983, Sol. Phys., 87, 135

Leenaarts, J., Pereira, T. M. D., Carlsson, M., Uitenbroek, H., \& De Pontieu, B. 2013a, ApJ, 772, 89

Leenaarts, J., Pereira, T. M. D., Carlsson, M., Uitenbroek, H., \& De Pontieu, B. 2013b, ApJ, 772, 90

López Ariste, A., Rayrole, J., \& Semel, M. 2000, A\&AS, 142, 137

Matsumoto, T., Kitai, R., Shibata, K., et al. 2008, PASJ, 60, 95

Mein, P., \& Mein, N. 1988, A\&A, 203, 162

Milkey, R. W., \& Mihalas, D. 1974, ApJ, 192, 769

Nelson, C. J., Doyle, J. G., Erdélyi, R., et al. 2013a, Sol. Phys., 283, 307

Nelson, C. J., Shelyag, S., Mathioudakis, M., et al. 2013b, ApJ, 779, 125

Nelson, C. J., Scullion, E. M., Doyle, J. G., Freij, N., \& Erdélyi, R. 2015, ApJ, 798, 19

Ni, L., Kliem, B., Lin, J., \& Wu, N. 2015, ApJ, 799, 79

Pariat, E., Aulanier, G., Schmieder, B., et al. 2004, ApJ, 614, 1099

Pariat, E., Schmieder, B., Berlicki, A., et al. 2007, A\&A, 473, 279

Pariat, E., Masson, S., \& Aulanier, G. 2009, ApJ, 701, 1911

Pereira, T. M. D., Leenaarts, J., De Pontieu, B., Carlsson, M., \& Uitenbroek, H. 2013, ApJ, 778, 143

Peter, H., Tian, H., Curdt, W., et al. 2014, Science, 346, 1255726

Rutten, R. J., Vissers, G. J. M., Rouppe van der Voort, L. H. M., Sütterlin, P., \&

Vitas, N. 2013, J. Phys. Conf. Ser., 440, 012007

Rybicki, G. B., \& Hummer, D. G. 1991, A\&A, 245, 171

Rybicki, G. B., \& Hummer, D. G. 1992, A\&A, 262, 209

Sandlin, G. D., Bartoe, J.-D. F., Brueckner, G. E., Tousey, R., \& Vanhoosier, M. E. 1986, ApJS, 61, 801

Schmieder, B., Rust, D. M., Georgoulis, M. K., Démoulin, P., \& Bernasconi, P. N. 2004, ApJ, 601, 530

Schmieder, B., Archontis, V., \& Pariat, E. 2014, Space Sci. Rev., 186, 227

Shine, R. A., \& Linsky, J. L. 1974, Sol. Phys., 39, 49

Socas-Navarro, H., Martínez Pillet, V., Elmore, D., et al. 2006, Sol. Phys., 235, 75

Staath, E., \& Lemaire, P. 1995, A\&A, 295, 517

Tian, H., Xu, Z., He, J., \& Madsen, C. 2016, ApJ, 824, 96

Vissers, G. J. M., Rouppe van der Voort, L. H. M., \& Rutten, R. J. 2013, ApJ, 774, 32

Vissers, G. J. M., Rouppe van der Voort, L. H. M., Rutten, R. J., Carlsson, M., \& De Pontieu, B. 2015, ApJ, 812, 11

Watanabe, H., Kitai, R., Okamoto, K., et al. 2008, ApJ, 684, 736

Watanabe, H., Vissers, G., Kitai, R., Rouppe van der Voort, L., \& Rutten, R. J. 2011, ApJ, 736, 71

Xu, X.-Y., Fang, C., Ding, M.-D., \& Gao, D.-H. 2011, RA\&A, 11, 225 